\title{
KINETICS OF THE HYDROGENATION OF 2,4-DINITROTOLUENE OVER A PALLADIUM ON ALUMINA CATALYST
}

\author{
E. J. MOLGA ${ }^{\dagger}$ and K. R. WESTERTERP ${ }^{*}$ \\ Chemical Reaction Engineering Laboratories, Faculty of Chemical Engineering, University of Twente, \\ P.O. Box 217, 7500 AE Enschede, The Netherlands
}

(Received 11 November 1990; accepted 18 June 1991)

\begin{abstract}
The chemistry and kinetics of the catalytic hydrogenation of 2,4-dinitrotoluene (2,4-DNT) over a palladium on alumina catalyst have been investigated. The catalyst consists of porous cylindrical pellets of $4.2 \times 4.2 \mathrm{~mm}$ in which a shell of Pd has penetrated to a depth of the order of $100 \mu \mathrm{m}$. The experiments have been performed in a three-phase batch reactor under isothermal and isobaric conditions. Two reactor temperatures of 308 and $345 \mathrm{~K}$ and a hydrogen pressure range $0.5-4 \mathrm{MPa}$ have been chosen. Employing a chromatographic method, analyses of the reaction mixtures have been carried out and the reaction pathway has been studied-three stable intermediates have been identified. We have concluded that for both types of catalyst, independent of the palladium carrier, the chemistry of the conversion of DNT into DAT is the same. Mass transfer experiments have shown that external mass transfer resistances can be neglected. However, internal diffusion limitations had to be taken into account. To study the intrinsic reaction kinetics, a series of measurements with a finely crushed catalyst of particle diameter lower than $40 \mu \mathrm{m}$ have been carried out. To describe the kinetics, a Langmuir-Hinshelwood model based on adsorption of hydrogen and organic species on different active sites has been applied. The kinetics parameters have been determined and compared with data for a Pd on carbon catalyst. The influence of the internal mass transfer resistances on the reaction rates has been quantitatively described with an effectiveness factor. At higher temperatures an additional series of experiments with pellets has been performed at $371 \mathrm{~K}$ in order to obtain an impression of the possibilities to extrapolate our correlations outside the experimental region. $\mathbf{A}$ good agreement between experimental and calculated hydrogen consumption rates is observed.
\end{abstract}

\section{INTRODUCTION}

In our laboratories studies of the catalytic hydrogenation of aromatic nitrocompounds have been performed and related by Westerterp et al. (1988), van Gelder et al. (1990a,- b) and Janssen et al. (1990a, b). The catalytic three-phase hydrogenation of 2,4-dinitrotoluene (DNT) over a $5 \%$ palladium on active carbon catalyst ( $\mathrm{Pd} / \mathrm{C})$ dissolved in methanol has been studied by Janssen et al. (1990a, b); they also presented a detailed literature survey. They have shown that the conversion of 2,4-DNT proceeds via a complex reaction pathway. A part from the end product 2,4-diaminotoluene (2,4-DAT), they found three stable intermediate products: 4-hydroxylamino-2nitrotoluene (4-HA-2-NT), 2-amino-4-nitrotoluene (2-A-4-NT) and 4-amino-2-nitrotoluene (4-A-2-NT). The reaction network consisting of parallel and consecutive pathways is shown in Fig. 1.

The effects of mass transfer resistances in the catalytic three-phase hydrogenation of aromatic nitrocompounds have been investigated experimentally by Yücelen (1984) as well as in other systems a.o. by Zwicky and Gut (1978). A significant influence of mass transfer limitations on the reaction rate and selectivity is reported.

\footnotetext{
'Permanent address: Institute of Chemical and Process Engineering. Warsaw Technical University, ul Warynskiego 1, 00-645 Warsaw, Poland.

'Author to whom correspondence should be addressed.
}

In this paper we present kinetic results obtained in a three-phase batch reactor for the hydrogenation of 2,4-DNT over a palladium on alumina catalyst. The aim of this work is to study the reaction kinetics on a pelletized catalyst, which can be used in packed bed reactors. In order to investigate the intrinsic reaction kinetics, also experiments with a crushed catalyst have been carried out.

\section{EXPERIMENTAL INSTALLATION AND PROCEDURE}

The experimental set-up is shown in Fig. 2. A special basket filled with catalyst pellets has been immersed into the liquid. A detailed drawing of the basket is presented in Fig. 3. A space of about $1 \mathrm{~mm}$ between the reactor wall and the basket as well as a Rushton type turbine mixer lead to a perfect mixing of both the gas and liquid. The crushed catalyst has been dispersed directly into the reaction liquid. The main apparatus dimensions and operating conditions are presented in Table 1.

The reactor system has been fully automated with a $\mu$ Mac-5000 controller connected to an Apple II $^{\circ}$ computer. The temperature control system consisted of calibrated chromel-alumel thermocouples, an Eurotherm electronic temperature controller as well as cooling and heating circuits. It was possible to operate the reactor under strictly isothermal conditions. The maximal deviations of the overall reactor temperature were never higher than $0.6^{\circ} \mathrm{C}$. The pressure measurement and regulation system has been 
E. J. Molga and K. R. Westertfrp
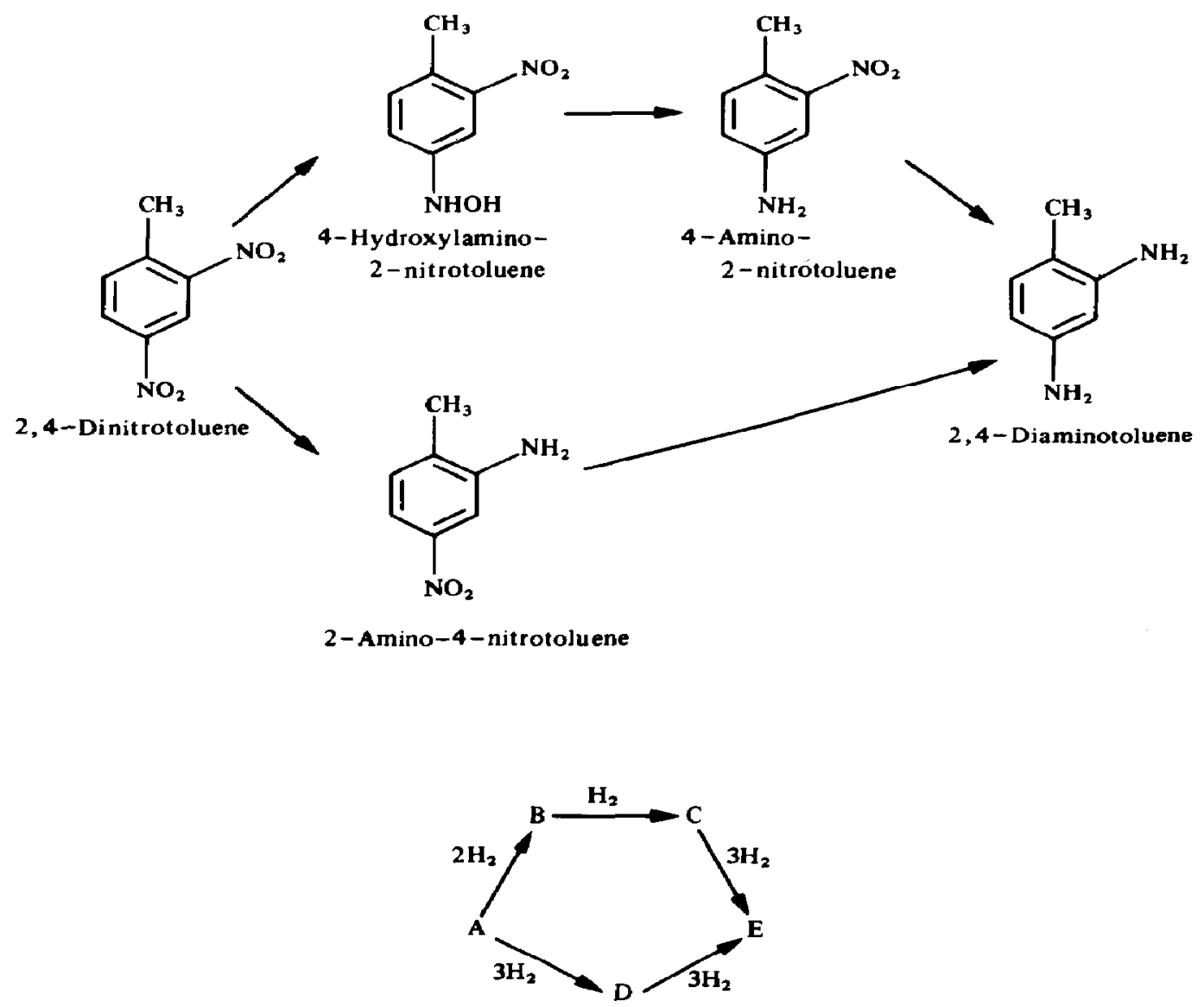

Fig. 1. Reaction scheme for the catalytic hydrogenation of 2,4-DNT.

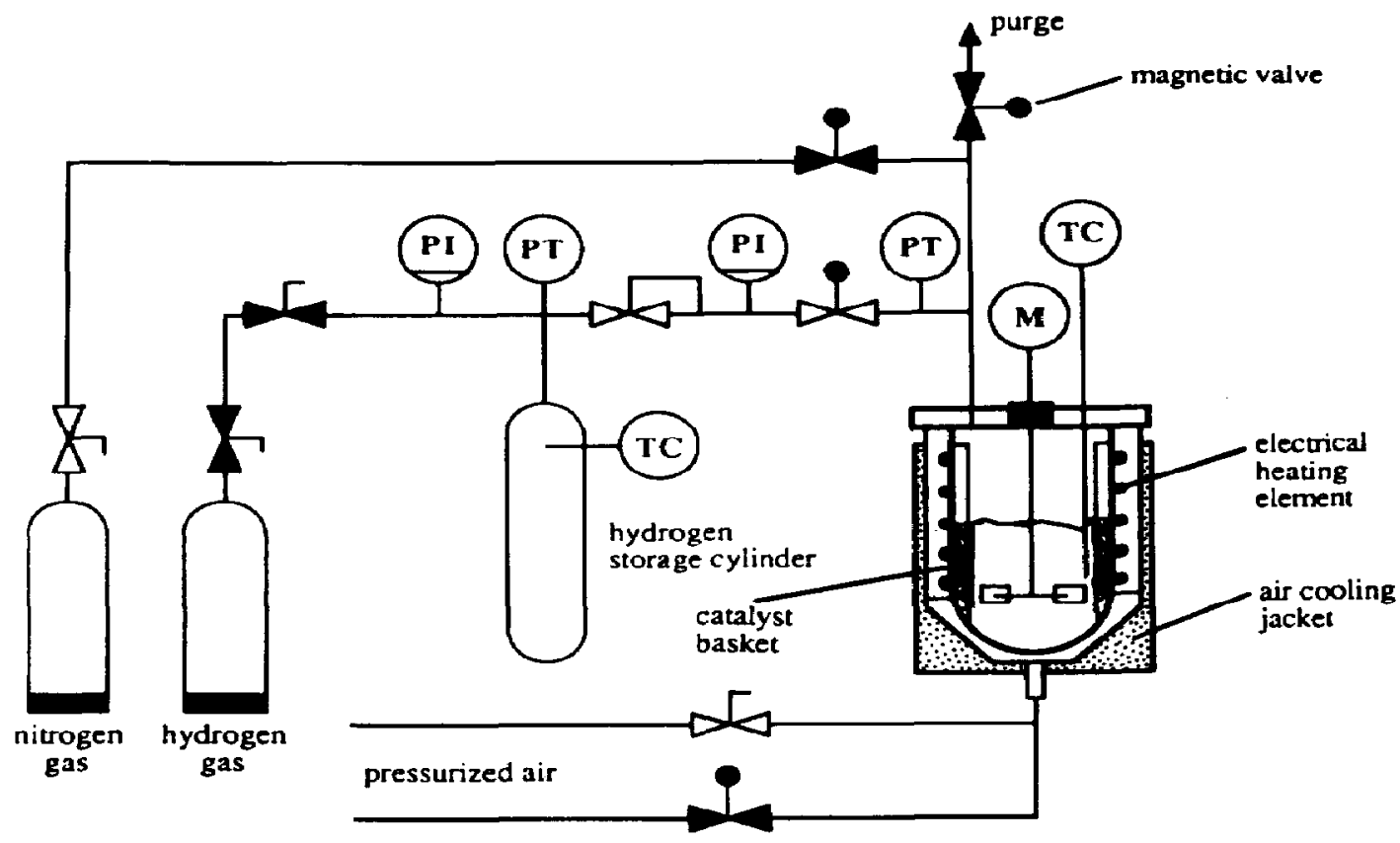

Fig. 2. Experimental installation: $\mathbf{M}=$ motor, $\mathbf{P I}=$ bourdon type pressure manometer, $\mathbf{P T}=$ electronic pressure transducer, $\mathrm{TC}=$ thermocouple. 
Table 1. Apparatus dimensions and operating conditions

Reactor diameter

Diameter of the agitator

Distance between agitator and reactor bottom

Reactor volume

Liquid-phase volume

Volume of the hydrogen storage cylinder

Reactor temperature

Pressure range in the reactor

Pressure range in the hydrogen storage cylinder Impeller speed
$75 \mathrm{~mm}$

$44 \mathrm{~mm}$

$40 \mathrm{~mm}$

$0.500 \mathrm{dm}^{3}$

$0.250 \mathrm{dm}^{3}$

$0.532 \mathrm{dm}^{3}$

308 and $345 \mathrm{~K}$

$0.5-4 \mathrm{MPa}$

5-10 MPa

25 rps

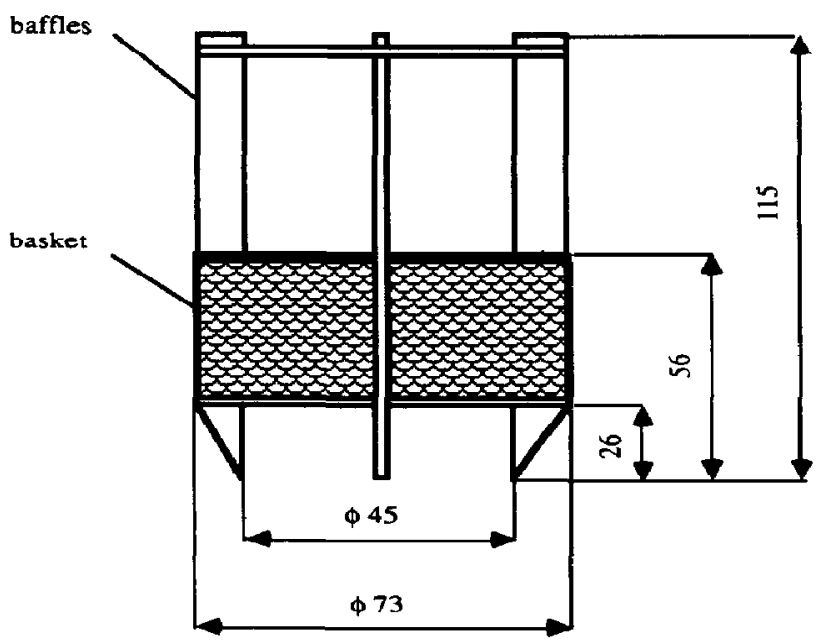

Fig. 3. The catalyst basket.

equipped with Bell and Howell pressure transducers and a Tescom pressure regulator as well as magnetic valves. The pressure transducers have been calibrated against high precision manometers. For each run the standard deviations of temperature and pressure were $0.2-0.3 \mathrm{~K}$ and $4-6 \mathrm{kPa}$, respectively.

The palladium on alumina catalyst is manufactured by Girdler Süd Chemie A.G. It contains $0.08 \mathrm{wt} \%$ of Pd and consists of $4.2 \times 4.2 \mathrm{~mm}$ cylindrical pellets of alumina covered with a thin layer of $P d$ in the outer shell of the pellets. In a separate series of experiments a crushed catalyst with a particle diameter not higher than $\mathbf{4 0} \mu \mathrm{m}$ has been used to determinc the intrinsic reaction kinetics, that is, without pore diffusion limitations. Hydrogen of Hoek Loos with a purity of higher than $99.9 \mathrm{vol} \%$ and 2,4-DNT supplied by Janssen Chimica with a purity of higher than $98 \%$ have been applied as reactants and methanol has been used as the solvent.

During the course of the reaction the temperature and pressure in the reactor as well as the temperature and pressure in the hydrogen storage cylinder are monitored and registered. The molar amount $n$ of hydrogen in the experimental system, in the reactor and also in the hydrogen storage cylinder have been calculated with $n=p_{\mathrm{H}_{2}} V / z R T$ where the compressibility factor $z$ has been estimated from data collected by Perry and Chilton (1974).
A correction for the amount of hydrogen dissolved in methanol at the beginning of the process has been made on the basis of the data of Radhakrishnan et al. (1983). A further correction for the methanol vapour pressure and for nitrogen gas pressure in the reactor have been made according to $p_{\mathrm{H}_{2}}=p_{R}-p_{C}$, where $p_{C}$ is the sum of the methanol vapour pressure and the pressure of the remaining nitrogen gas. Discrepancies between the experimental hydrogen consumption and the theoretical hydrogen demand were never greater than $2.5 \%$. This theoretical demand has been calculated on the basis of the initial amount of 2,4-DNT assuming that the only end product of the hydrogenation is 2,4-DAT. In an experiment where the liquid phase concentrations have been determined, the reaction was stopped at a certain hydrogen conversion, whereafter a sample of the liquid was taken and analysed immediately with a gas chromatograph. After that the reactor was emptied and loaded again with fresh catalyst and fresh reactant. A flame ionization detector in a Varian 3300 gas chromatograph equipped with $6 \mathrm{ft} \times 1 / 8$ " Tenax 60/80 mesh column and a HP 3392A integrator were employed. For calibration purposes 4-hydroxylamino-2-nitrotoluene (4HA-2NT) was synthesized by ourselves; the other organic compounds were purchased from Janssen Chimica.

\section{KINETIC MODELLING}

The basic principles of complex reaction modelling in three-phase systems are discussed a.o. by Westerterp et al. (1987) and a detailed description of the hydrogenation of 2,4-DNT over a Pd/C catalyst can be found in Janssen et al. (1990b). In our case, again, a Langmuir-Hinshelwood model has been applied under the following assumptions:

-Adsorption of hydrogen and organic compounds occurs at different active sites of catalyst.

-Organic compounds compete with each other for adsorption.

-An adsorption equilibrium is established under all conditions.

According to these assumptions the rate of the conversion of compound $i$ to $j$ can be represented by

$$
R_{i j}=c_{c a t} k_{i j} \theta_{i} \theta_{\mathrm{H}}
$$


where $\theta_{i}$ and $\theta_{\mathrm{H}}$ are the fractional catalyst coverages by substrate $i$ and hydrogen, respectively, $k_{i j}$ is the reaction rate constant per unit mass of the catalyst and $c_{c a t}$ is the catalyst concentration per unit of liquid volume $m_{\text {eat }} / V_{R L}$. The fractional coverage by hydrogen $\theta_{\mathbf{H}}$ is given by the equation

$$
\theta_{\mathrm{H}}=\frac{K_{\mathrm{H}}\left(p_{\mathrm{H}_{2}} / R T\right)^{n}}{1+K_{\mathrm{H}}\left(p_{\mathrm{H}_{2}} / R T\right)^{n}}
$$

where $n$ equals 1 for molecular hydrogen adsorption and 0.5 for dissociative adsorption, and $p_{\mathrm{H}_{2}}$ is the partial pressure of hydrogen. The chemisorption constant $K_{H}$ is a function of the true adsorption equilibrium constant and the solubility of hydrogen in methanol. The fractional catalyst coverage for the $i$ th organic reactant is given by

$$
\theta_{i}=\frac{K_{i} c_{i}}{1+K_{i} c_{i}+\sum_{j \neq i} K_{j} c_{j}}
$$

where $K_{i}$ is the chemisorption constant and $c_{i}$ is the concentration of substrate $i$ in the liquid phase. For the reaction scheme shown in Fig. 1 and applying eqs (1)-(3), the consumption rate of component $A$ can be derived to be

$$
-\frac{\mathrm{d} c_{A}}{\mathrm{~d} t}=c_{\mathrm{cat}}\left(k_{A B}+k_{A D}\right) \theta_{\mathrm{H}} \frac{K_{A} c_{A}}{1+K_{A} c_{A}+\sum_{j \neq A} K_{j} c_{j}} .
$$

Introducing a reduced time

$$
t_{\mathrm{red}}=\frac{t m_{\mathrm{cat}}}{n_{\mathrm{DNT} 0}}=\frac{t c_{\mathrm{cat}}}{c_{A 0}}
$$

and dimensionless concentration for the organic species $C_{i}=\mathrm{c}_{i} / c_{A 0}$ we obtain

$$
-\frac{\mathrm{d} C_{A}}{\mathrm{~d} t_{\mathrm{red}}}=\frac{\theta_{\mathrm{H}}\left(k_{A B}+k_{A D}\right) C_{A}}{M}
$$

where

$$
\begin{aligned}
M= & \frac{1}{K_{A} c_{A 0}}+C_{A}+\frac{K_{B} C_{B}}{K_{A}}+\frac{K_{C} C_{C}}{K_{A}} \\
& +\frac{K_{D} C_{D}}{K_{A}}+\frac{K_{E} C_{E}}{K_{A}}
\end{aligned}
$$

For the remaining reactants the following set of differential equations can been derived:

$$
\begin{aligned}
& \frac{\mathrm{d} C_{B}}{\mathrm{~d} t_{\text {red }}}=\frac{\theta_{\mathrm{H}}\left(k_{A B} K_{A} C_{A} / K_{A}-k_{B C} K_{B} C_{B} / K_{A}\right)}{M} \\
& \frac{\mathrm{d} C_{C}}{\mathrm{~d} t_{\text {red }}}=\frac{\theta_{\mathrm{H}}\left(k_{B C} K_{B} C_{B} / K_{A}-k_{C E} K_{C} C_{C} / K_{A}\right)}{M} \\
& \frac{\mathrm{d} C_{D}}{\mathrm{~d} t_{\text {red }}}=\frac{\theta_{\mathrm{H}}\left(k_{A D} K_{A} C_{A} / K_{A}-k_{\mathrm{DE}} K_{D} C_{D} / K_{A}\right)}{M} .
\end{aligned}
$$

From a material balance we can obtain the concentration of the final product DAT:

$$
C_{E}=1-C_{A}-C_{B}-C_{C}-C_{D} .
$$

\section{EXPERIMENTAL RISULTS}

The experiments performed can be classified into the following groups:

Concentration versus time experiments to determine the reaction pathway.

- Mass transfer experiments to study external and internal mass transfer limitations.

-Experiments with finely crushed catalyst to determine the intrinsic reaction kinetics.

-Experiments with catalyst pellets to investigate the influence of internal mass transfer limitations.

\subsection{Concentration versus time measurements}

By chromatographic analysis three stable intermediates have been found in larger concentrations

Table 2. Concentration versus time measurements

(a) $T=308 \mathrm{~K}, p_{\mathrm{H}_{2}}=1.0 \mathrm{MPa}, c_{10}=219 \mathrm{~mol} \mathrm{~m}^{-3}$, pellets

\begin{tabular}{lrlllllll}
\hline Run & $t_{\text {red }}{ }^{9}$ & $\zeta_{\mathrm{H}_{2}, \text { exp }}$ & $C_{A}$ & $C_{B}$ & $C_{C}$ & $C_{\mathrm{D}}$ & $C_{E}$ & $\zeta_{\mathrm{H}_{2}, \text { En1 }}$ \\
\hline A1 & 319 & 0.106 & 0.765 & 0.211 & 0.008 & 0.013 & 0.003 & 0.116 \\
A2 & 661 & 0.224 & 0.464 & 0.336 & 0.101 & 0.082 & 0.017 & 0.232 \\
A3 & 953 & 0.305 & 0.325 & 0.425 & 0.147 & 0.052 & 0.051 & 0.320 \\
A4 & 1420 & 0.412 & 0.136 & 0.377 & 0.276 & 0.119 & 0.100 & 0.423 \\
A5 & 1881 & 0.513 & 0.037 & 0.390 & 0.336 & 0.121 & 0.151 & 0.510 \\
A6 & 3069 & 0.692 & 0.002 & 0.116 & 0.458 & 0.099 & 0.348 & 0.665 \\
A7 & 4634 & 0.864 & 0.000 & 0.000 & 0.334 & 0.060 & 0.572 & 0.769 \\
A8 & 5733 & 0.976 & 0.004 & 0.000 & 0.166 & 0.044 & 0.829 & 0.933
\end{tabular}

(b) $T=345 \mathrm{~K}, P_{\mathrm{H}_{2}}=1.0 \mathrm{MPa}, c_{A O}=211 \mathrm{~mol} \mathrm{~m}^{-3}$, pellets

\begin{tabular}{lrlllllll}
\hline A9 & 90 & 0.125 & 0.751 & 0.151 & 0.071 & 0.027 & 0.035 & 0.131 \\
A10 & 249 & 0.319 & 0.399 & 0.256 & 0.198 & 0.087 & 0.081 & 0.309 \\
A11 & 478 & 0.533 & 0.133 & 0.067 & 0.418 & 0.157 & 0.215 & 0.525 \\
A12 & 786 & 0.742 & 0.026 & 0.000 & 0.416 & 0.126 & 0.460 & 0.722 \\
A13 & 1335 & 0.904 & 0.000 & 0.000 & 0.258 & 0.072 & 0.760 & 0.896 \\
\hline
\end{tabular}

\footnotetext{
${ }^{\dagger}$ Units s kg cat mol ${ }^{-1}$ DNT.
} 
in the reaction mixture: 4-hydroxylamino-2-nitrotoluene (4-HA-2-NT), 4-amino-2-nitrotoluene (4-A-2NT) and 2-amino-4-nitrotoluene (2-A-4-NT). The results of the concentration measurements for catalyst pellets are presented in Table 2 as a function of the reduced time and the hydrogen coversion $\zeta_{\mathrm{H}_{2}}$. The hydrogen conversion $\zeta_{\mathrm{H}_{3}}$ is defined as the ratio of the amount of hydrogen actually consumed over the amount of hydrogen consumed for a complete conversion of DNT to DAT:

$$
\zeta_{\mathbf{H}_{2}}(t)=\frac{n_{\mathbf{H}_{2}}^{0}-n_{\mathrm{H}_{2}}(t)}{n_{\mathbf{H}_{2}}^{0}-n_{\mathbf{H}_{2}}^{\infty}}
$$

The concentrations and conversions can be checked by comparing the hydrogen conversion values $\zeta_{\mathrm{H}_{2} \text {, cal }}$ calculated according to

$$
\begin{aligned}
\zeta_{\mathrm{H}_{2} \text {, eal }} & =\frac{2 c_{B}+3 c_{C}+3 c_{D}+6 c_{E}}{6 c_{A 0}} \\
& =\frac{1}{3} C_{B}+\frac{1}{2} C_{C}+\frac{1}{2} C_{D}+C_{E}
\end{aligned}
$$

with the values of $\zeta_{\mathrm{H}_{2} \text {.exp }}$ as determined from the independent experimental hydrogen consumption measurements. This comparison is shown in the parity plot of Fig. 4. The concentration profiles obtained lead us to the conclusion that the reaction pathway as elaborated by Janssen et al. (1990a) for the hydrogenation of 2,4-DNT over a $\mathrm{Pd} / \mathrm{C}$ catalyst is also valid when a $\mathrm{Pd}$ on $\mathrm{Al}_{2} \mathrm{O}_{3}$ catalyst is used. The reaction scheme shown in Fig. 1 indicates that an orthonitro group is converted directly to the corresponding aminogroup whereas on hydrogenating the paranitro group, first 4-hydroxylamine-2-nitrotoluene is formed.

\subsection{Initial reaction rate}

Overall reaction rates can be estimated directly from the hydrogen conversion versus time measurements. To compare the hydrogenation results in different experiments, a reduced reaction time $t_{\text {red }}$ as defined previously has been introduced to take into account differences in the initial amount of DNT and in the amount of catalyst added. The hydrogen conversion data obtained for catalyst pellets at $308 \mathrm{~K}$ are shown in Fig. 5. The influence of the hydrogen pressure on the reaction rate is evident although the hydrogen consumption rate does not increase proportionally with the hydrogen pressure. Characteristic instabilities of the hydrogen consumption rates immediately after starting the reaction have been observed; it takes approximately $200 \mathrm{~s}$ before the reaction conditions are stabilized. This initial period has been omitted in the data evaluation. Observing the conversion data shown in Fig. 6, we see that in the beginning the reaction rate is zero-order DNT in the conversion range up to 0.20 . Overall initial reaction rates, according to $r_{\mathrm{O}}=\Delta \zeta_{\mathrm{H}_{2}} / \Delta t$, are obtained by a linear regression of the hydrogen conversion versus time data. A reduced initial rate for the hydrogen consumption can be calculated according to

$$
r_{\mathrm{O}, \mathrm{H}}=\frac{6 r_{0} c_{\text {DNTO }}}{c_{\text {cat }}}
$$

According to the reaction scheme this initial rate is the sum of two reactions of DNT to 4-HA-2-NT and to 2-A-4-NT. The rate constants of these two reactions can be influenced differently by temperature. Conditions chosen and the results for the experiments carried out with catalyst pellets are given in Table 3 .

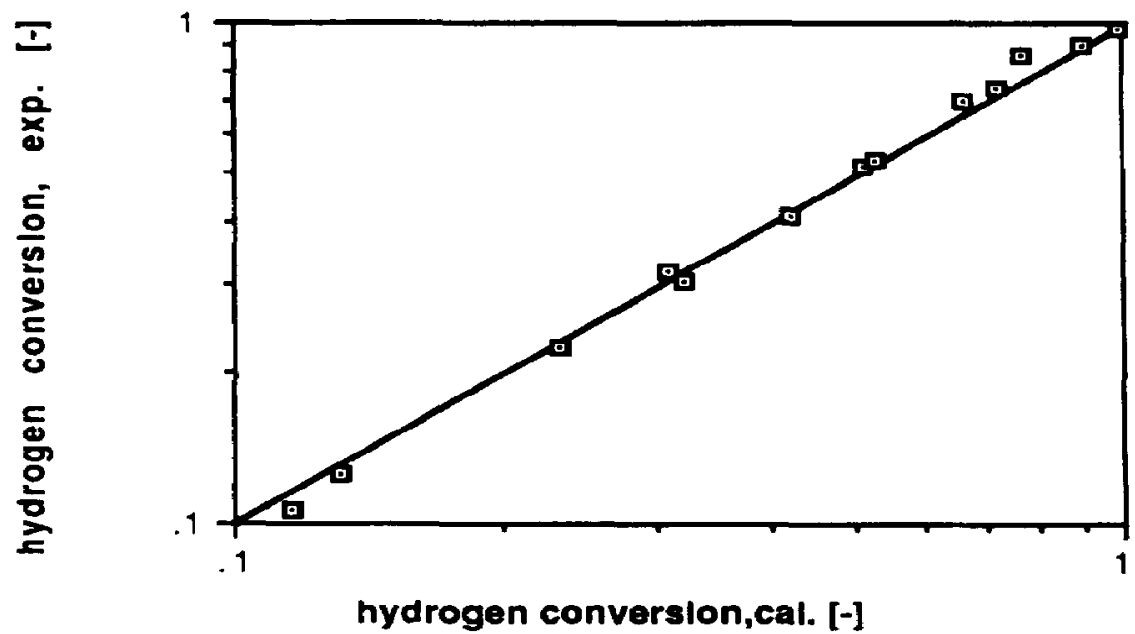

Fig. 4. Comparison of the experimental hydrogen conversions with those obtained from the concentration measurements. 


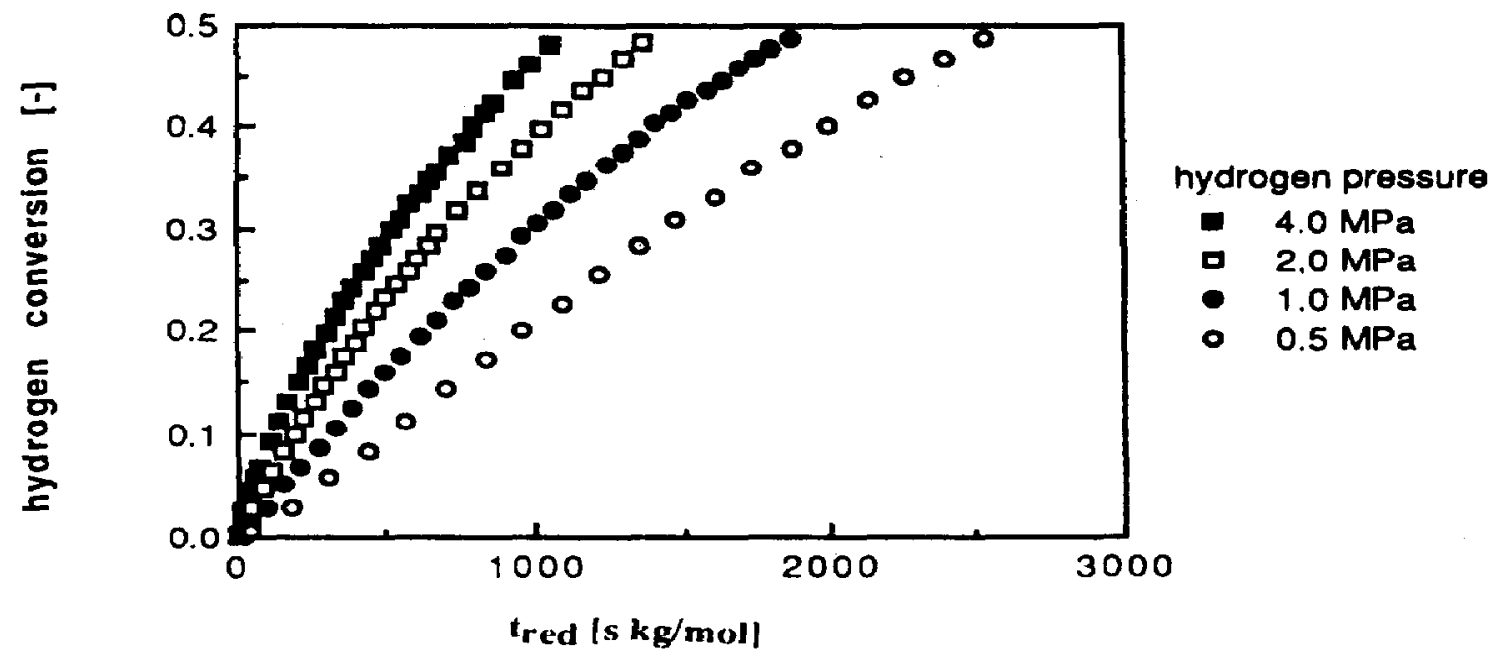

Fig. 5. Conversion data obtained for catalyst pellets at $308 \mathrm{~K}$. Runs P1-P4 as in Table 3.

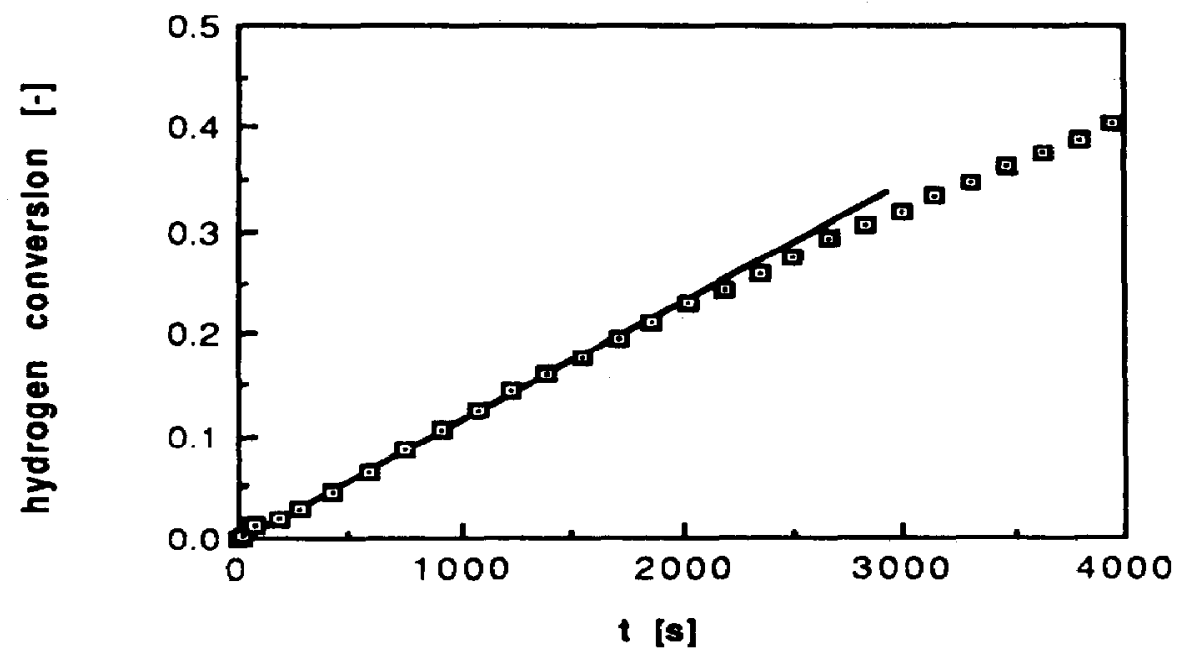

Fig. 6. Conversion of hydrogen in the initial region. $T=308 \mathrm{~K}, p_{\mathrm{H}_{2}}=1.0 \mathrm{MPa}, c_{A 0}=219 \mathrm{~mol} \mathrm{~m}^{-3}$, $m_{\text {cat }}=20 \mathrm{~g}$.

Table 3. Hydrogen consumption rates for pellets of catalyst

\begin{tabular}{|c|c|c|c|c|c|c|c|}
\hline Run & $\begin{array}{c}T \\
(\mathbf{K})\end{array}$ & $\begin{array}{c}p_{\mathrm{H}_{2}} \\
(\mathrm{kPa})\end{array}$ & $\begin{array}{c}m_{\mathrm{cat}} \\
(\mathrm{g})\end{array}$ & $\begin{array}{c}m_{\mathrm{O}, \mathrm{DNT}} \\
\quad(\mathrm{g})\end{array}$ & $\begin{array}{c}r_{0} \times 10^{3} \\
\left(s^{-1}\right)\end{array}$ & $\begin{array}{c}r_{0, H} \times 10^{3} \\
\left(\mathrm{~mol} \mathrm{~kg}^{-1} \mathrm{~s}^{-1}\right)\end{array}$ & $\begin{array}{c}c_{\mathbf{H}_{2,8}} \\
\left.\operatorname{mol~m}^{-3}\right)\end{array}$ \\
\hline $\begin{array}{l}\text { P1 } \\
\text { P2 } \\
\text { P3 } \\
\text { P4 }\end{array}$ & $\begin{array}{l}308.7 \\
308.6 \\
308.7 \\
308.7\end{array}$ & $\begin{array}{r}496 \\
993 \\
1995 \\
3938\end{array}$ & $\begin{array}{l}19.79 \\
19.56 \\
19.71 \\
19.43\end{array}$ & $\begin{array}{l}8.82 \\
9.96 \\
8.24 \\
8.93\end{array}$ & $\begin{array}{l}0.089 \\
0.111 \\
0.197 \\
0.269\end{array}$ & $\begin{array}{l}1.34 \\
1.79 \\
2.86 \\
3.86\end{array}$ & $\begin{array}{r}193 \\
387 \\
778 \\
1535\end{array}$ \\
\hline $\begin{array}{l}\text { P5 } \\
\text { P6 } \\
\text { P7 } \\
\text { P8 }\end{array}$ & $\begin{array}{l}345.7 \\
345.6 \\
345.6 \\
345.7\end{array}$ & $\begin{array}{r}501 \\
1001 \\
1992 \\
3986\end{array}$ & $\begin{array}{l}19.60 \\
19.67 \\
19.21 \\
18.90\end{array}$ & $\begin{array}{l}8.94 \\
9.62 \\
8.31 \\
8.66\end{array}$ & $\begin{array}{l}0.282 \\
0.465 \\
0.912 \\
1.173\end{array}$ & $\begin{array}{c}4.23 \\
7.80 \\
12.6 \\
17.8\end{array}$ & $\begin{array}{r}175 \\
349 \\
693 \\
1387\end{array}$ \\
\hline
\end{tabular}




\subsection{Check for external mass transfer limitations}

The contribution of every mass transfer resistance as well as the contribution of a kinetic resistance to the overall hydrogen consumption rate can be described as follows:

$$
\begin{aligned}
\frac{c_{\mathrm{H}_{2} e L}}{J_{\mathrm{H}_{2}} a_{G L}}= & \frac{m}{k_{G} a_{G L}}+\frac{1}{k_{L} a_{G L}}+\frac{1}{k_{L S} a_{S}} \\
& +\frac{1}{\left(R_{\mathrm{H}_{2}}^{\prime \prime} / c_{\mathrm{H}_{2} i S}\right) a_{S}}
\end{aligned}
$$

where $c_{\mathrm{H}_{2} \text { eL }}=m p_{\mathrm{H}_{2}} / R T$ is the equilibrium concentration of hydrogen in the liquid phase, $m$ the solubility constant and $k_{G}, k_{L}$ and $k_{L S}$ the mass transfer coefficients in the gas phase and in the liquid phase at the gas-liquid interface and the liquid-solid interface, respectively. The symbols $a_{G L}$ and $a_{s}$ denote the gas-liquid and liquid-solid specific interfacial areas, $\boldsymbol{R}_{\mathrm{H}_{2}}^{\prime \prime}$ the reaction rate per unit external surface area of the catalyst and $c_{\mathrm{H}_{2} \text { is }}$ the concentration of hydrogen at the catalyst surface. In eq. (15) each individual contribution is given as the time constant of that individual step. Contributions are negligible if their time constants are very small compared to the other ones. The individual resistances will be disscused at $345 \mathrm{~K}$ and at the start of the reaction where the reaction rates are highest. The reciprocal absorption rate per unit of equilibrium concentration in the liquid phase in the initial period is given by $c_{\mathrm{H}_{2} e L} / J_{\mathrm{H}_{2}} a_{G L}$. The solubility coefficient $m$ at $345 \mathrm{~K}$ is equal to 0.136 as estimated from data of Radhakrishnan et al. (1983). The overall initial transfer rate of the hydrogen $J_{\mathbf{H}_{2}} a_{G L}$ per unit volume of the liquid can be calculated from

$$
J_{\mathrm{H}_{2}} a_{G L}=\frac{6 r_{0} n_{\mathrm{DNTO}}}{V_{\mathrm{LR}}}
$$

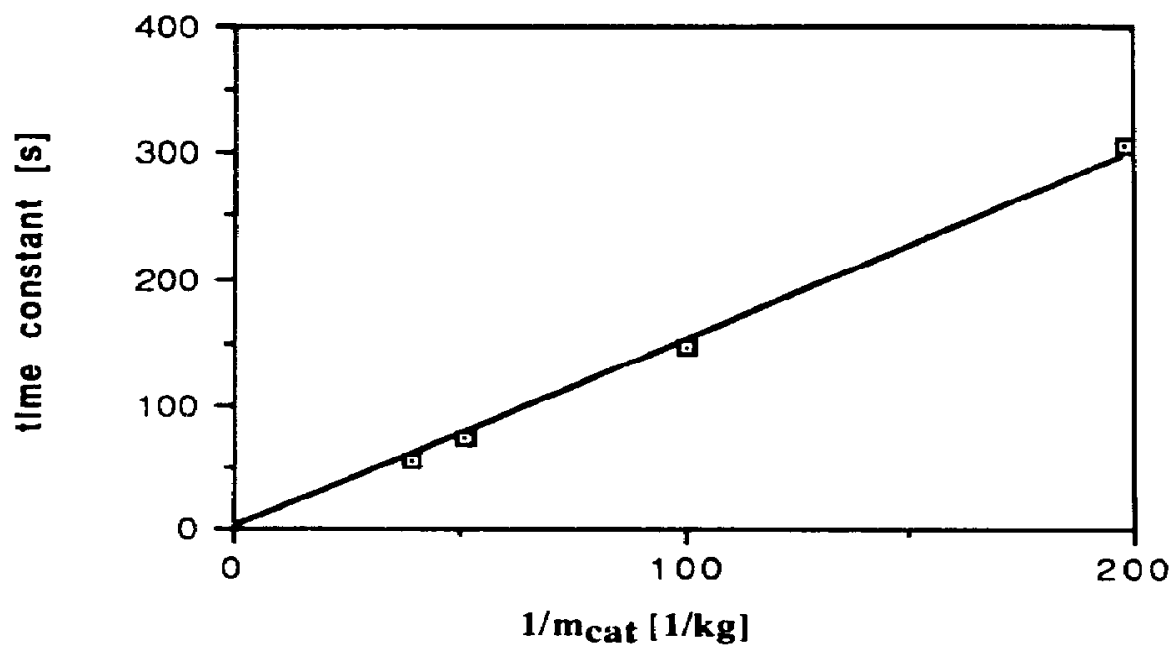

Fig. 7. Time constants $c_{\mathrm{H}_{2} \text { L }} / J_{\mathrm{H}_{2}} a_{G L}$ as a function of the catalyst loading. $T=345 \mathrm{~K}, p_{\mathrm{H}_{2}}=0.5 \mathrm{MPa}$,
In our experiments the values of $c_{\mathrm{H}_{2} e L} / J_{\mathrm{H}_{2}} a_{G L}$ varied from $72 \mathrm{~s}$ in run $\mathrm{P} 5$ to $509 \mathrm{~s}$ in run P4. The lowest value is found at $345 \mathrm{~K}$ and $0.5 \mathrm{MPa}$.

The time constant for the mass transfer through the gas-phase boundary, $m / k_{G} a_{G L}$, at $345 \mathrm{~K}$ has been estimated to be not higher than $1 \mathrm{~s}$, because $k_{G}$ is not lower than $0.001 \mathrm{~ms}^{-1}$ and $a_{G L}$ is roughly $1000 \mathrm{~m}^{2} \mathrm{~m}^{-3}$ liquid.

For the mass transfer through the liquid-side boundary the order of magnitiude of the time constant, $1 / k_{L} a_{G L}$, is about $2 \mathrm{~s}$ taking $k_{L}$ at $5 \times 10^{-4}$ $\mathrm{ms}^{-1}$. We also have checked its significance by varying the catalyst loading as shown in Fig. 7, where the time constants $c_{\mathrm{H}_{2} \text { eL }} / J_{\mathrm{H}_{2}} a_{G L}$ are plotted as a function of the reciprocal of the amount of catalyst. The straight line going through the origin shows that gas-liquid mass transfer resistances can be neglected. In Table 4 the results and the conditions are listed.

The liquid-side mass transfer coefficient $k_{L S}$ of the particle we estimate under our experimental conditions to be $0.004 \mathrm{~m} / \mathrm{s}$ and $a_{s}$ is $40 \mathrm{~m}^{2} \mathrm{~m}^{-3}$. So the time constant $1 / k_{L S} a_{S}$ amounts to about $6 \mathrm{~s}$ and its contribution to the total time constant $c_{\mathrm{H}_{2} e L} / J_{\mathrm{H}_{2}} a_{G L}$ never exceeds $10 \%$.

The values of the mass transfer coefficients $k_{G}, k_{L}$ and $k_{L S}$ as well as the specific interfacial $a_{G L}$ have been determined according to correlations given in Westerterp et al. (1987) and Ramachandran and Chaudari (1983). To check experimentally the significance of the external mass transfer resistances, the influence of the agitation speed on the overall hydrogen conversion has been investigated. Results shown in Table 4 and in Fig. 8 lead us to the conclusion that at the chosen experimental condition and for agitation rates higher than $20 \mathrm{~s}^{-1}$ the liquid phase is saturated with hydrogen and that the external mass transfer resistances can be neglected.

\footnotetext{
Fig. 7. Time constants $c_{\mathrm{H}_{2} e L} / J_{\mathrm{H}_{2}} a_{G L}$ as $\mathrm{mol} \mathrm{m}^{-3}$.
} 
Table 4. External mass transfer limitation tests for pellets

\begin{tabular}{|c|c|c|c|c|c|c|c|}
\hline \multicolumn{8}{|c|}{ (a) Catalyst loading, $c_{A 0}=196 \mathrm{~mol} \mathrm{~m}^{-3}$} \\
\hline Run & $\begin{array}{c}T \\
(\mathbf{K})\end{array}$ & $\begin{array}{c}P_{\mathbf{H}_{x}} \\
(\mathbf{k P a})\end{array}$ & $\begin{array}{c}m_{\mathrm{cat}} \\
(\mathrm{g})\end{array}$ & $\begin{array}{c}r_{0} \times 10^{3} \\
\left(\mathrm{~s}^{-1}\right)\end{array}$ & $\begin{array}{c}r_{0 . \mathrm{H}} \times 10^{3} \\
\left(\mathrm{~mol} \mathrm{~kg} \mathrm{k}^{-1} \mathrm{~s}^{-1}\right)\end{array}$ & 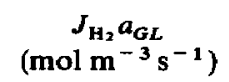 & $\begin{array}{c}c_{\mathrm{H}_{2} \in L} / J_{\mathrm{H}_{2}} a_{G L} \\
\text { (s) }\end{array}$ \\
\hline $\begin{array}{l}\text { M1 } \\
\text { M2 } \\
\text { M3 } \\
\text { M4 }\end{array}$ & $\begin{array}{l}345.4 \\
345.6 \\
345.4 \\
345.4\end{array}$ & $\begin{array}{l}485 \\
501 \\
491 \\
494\end{array}$ & $\begin{array}{r}25.34 \\
19.60 \\
9.99 \\
5.05\end{array}$ & $\begin{array}{l}0.373 \\
0.282 \\
0.134 \\
0.062\end{array}$ & $\begin{array}{l}4.35 \\
4.23 \\
4.04 \\
3.80\end{array}$ & $\begin{array}{l}0.441 \\
0.331 \\
0.162 \\
0.078\end{array}$ & $\begin{array}{r}54 \\
72 \\
147 \\
305\end{array}$ \\
\hline \multicolumn{8}{|c|}{ (b) Stirring rate, $m_{\mathrm{cat}}=20 \mathrm{~g}, c_{A 0}=218 \mathrm{~mol} \mathrm{~m}^{-3}$} \\
\hline Run & $\begin{array}{c}T \\
(\mathbf{K})\end{array}$ & $\underset{(\mathbf{k P a})}{p_{\mathrm{H}_{2}}}$ & $\begin{array}{c}N \\
(\mathbf{r p s})\end{array}$ & $\begin{array}{c}r_{0} \times 10^{3} \\
\left(s^{-1}\right)\end{array}$ & $\begin{array}{c}r_{\mathrm{O} . \mathrm{H}} \times 10^{3} \\
\left(\mathrm{~mol} \mathrm{~kg}{ }^{-1} \text { cat s}\right.\end{array}$ & & \\
\hline $\begin{array}{l}\text { M5 } \\
\text { M6 } \\
\text { M7 } \\
\text { M8 } \\
\text { M9 } \\
\text { M10 }\end{array}$ & $\begin{array}{l}345.4 \\
345.4 \\
345.6 \\
345.5 \\
345.4 \\
345.5\end{array}$ & $\begin{array}{l}502 \\
507 \\
501 \\
508 \\
503 \\
504\end{array}$ & $\begin{array}{l}35 \\
30 \\
25 \\
20 \\
15 \\
10\end{array}$ & $\begin{array}{l}0.289 \\
0.278 \\
0.282 \\
0.280 \\
0.243 \\
0.196\end{array}$ & $\begin{array}{l}4.37 \\
4.34 \\
4.23 \\
4.08 \\
3.77 \\
3.06\end{array}$ & & \\
\hline
\end{tabular}

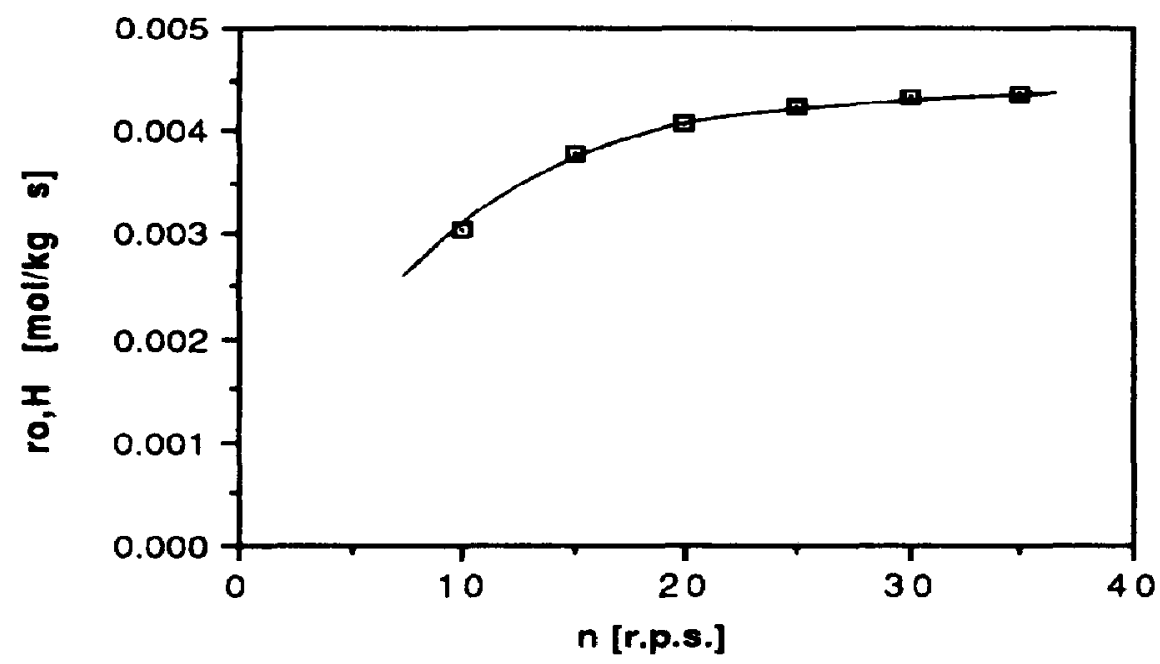

Fig. 8. Influence of the stirring rate on the initial reaction rate. $T=345 \mathrm{~K}, p_{\mathrm{H}_{2}}=0.5 \mathrm{MPa}$, $c_{A O}=196 \mathrm{~mol} \mathrm{~m}^{-3}, m_{\text {eat }}=20 \mathrm{~g}$.

4.4. Check for internal mass transfer limitations

Our catalyst is a shell catalyst and the penetration depth is expected to be of the order of $100 \mu \mathrm{m}$. Whether diffusion limitations play a role has to be checked experimentally. We have crushed and sieved five samples of the catalyst pellets to determine the particle size distribution and average particle size. Results are compared in Table 5 and plotted in Fig. 9-the initial reaction rate depends on the degree of crushing. The degree of crushing in Table 5 has

Table 5. Results of the internal diffusion limitations experiments

\begin{tabular}{lcccc}
\hline Run & $\begin{array}{c}T \\
(\mathrm{~K})\end{array}$ & $\begin{array}{c}\mathbf{P}_{\mathrm{H}_{2}} \\
(\mathbf{k P a})\end{array}$ & $\begin{array}{c}\boldsymbol{r}_{\mathrm{O}, \mathrm{H}} \times 10^{3} \\
\left(\mathrm{~mol} \mathrm{~kg}^{-1} \mathrm{~s}^{-1}\right)\end{array}$ & $\begin{array}{c}d_{p} \\
(\mu \mathrm{m})\end{array}$ \\
\hline M11 & 345.7 & 501 & 4.23 & Pellets $4.2 \times 4.2 \mathrm{~mm}$ \\
M12 & 345.4 & 497 & 7.32 & 535 \\
M13 & 345.3 & 511 & 14.7 & 110 \\
M14 & 345.3 & 494 & 15.4 & 65 \\
M15 & 345.4 & 492 & 17.5 & 50 \\
M16 & 345.6 & 497 & 17.6 & 40 \\
\hline
\end{tabular}




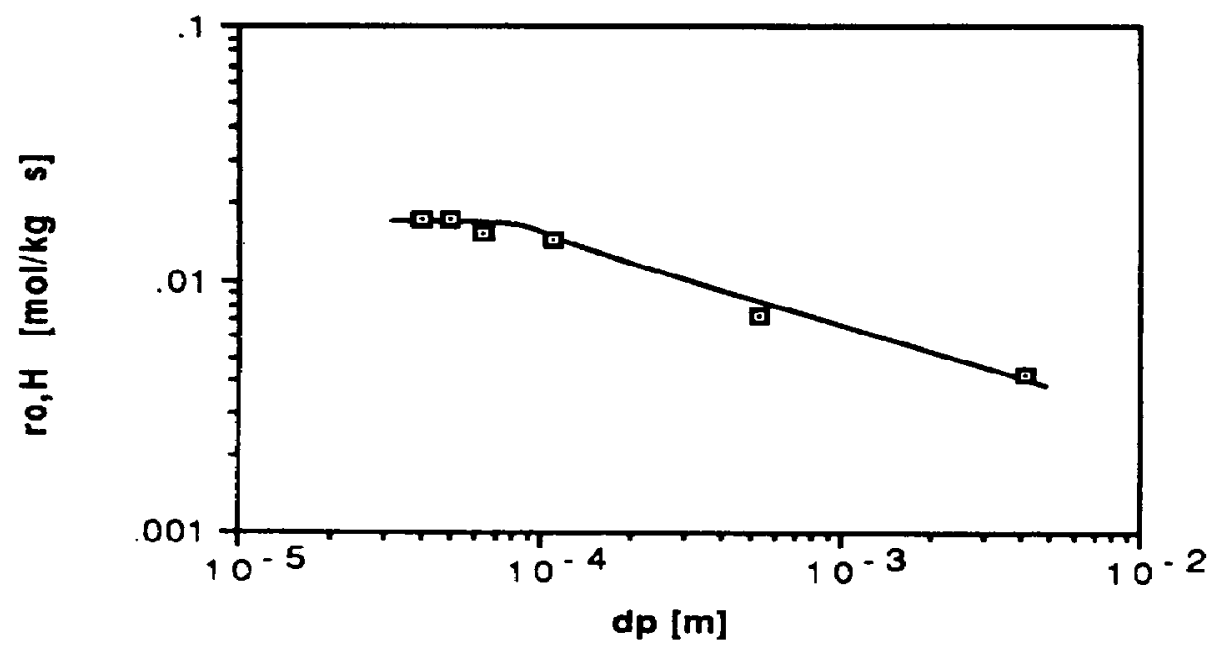

Fig. 9. Initial hydrogen consumption rates as a function of an averaged catalyst particle diameter. $T=345 \mathrm{~K}, p_{\mathrm{H}_{2}}=0.5 \mathrm{MPa}, c_{\mathrm{AO}}=196 \mathrm{molm}^{-3}, m_{\mathrm{cat}}=20 \mathrm{~g}$

been expressed as a kind of average particle diameter obtained from the mass fractions collected on the sieves in a sieve analysis and a particle diameter taken as the square root of the product of subsequent sieve diameters. We can observe that internal diffusion resistances should be taken into account for the catalyst pellets and for a crushed catalyst with particle sizes higher than $60 \mu \mathrm{m}$ for the reaction condition of Table 5.

To study the intrinsic reaction kinetics, a separate series of experiments has been carried out with finely crushed catalyst with a particle size not higher than $40 \mu \mathrm{m}$. The conditions chosen and the initial reaction rates, determined in the same way as for pellets, are given in Table 6 . By changing the agitation rates from 15 to 30 rps at $345 \mathrm{~K}$ and at a hydrogen pressure of $0.5 \mathrm{MPa}$, we have verified that external mass transfer resistances for this series of experiments can be neglected.

\section{ESTIMATION OF THE MODEL PARAMETERS}

\subsection{Intrinsic reaction kinetics}

Parameters of the model presented in Section 3 have been estimated from finely crushed catalyst data, as listed in Table 6. The Simusolv programModelling and Simulation Software: copyright the Dow Chemical Co.- which is based on the maximum-likelihood principle has been employed. The experimental data of the hydrogen conversion as well as the concentration data have been used. Preliminary calculations performed indicated that the product $K_{A} c_{A 0}$ under all conditions was much higher than unity, so the first term in eq. (7) could be eliminated. The results for each temperature separately are listed in Table 7. The reduced kinetic parameters $q_{i j}=k_{i j} /$ $k_{A B}$ and reduced chemisorption constants $Q_{i}-K_{i} / K_{A}$ as well as the values of the product $k_{A B} \theta_{\mathrm{H}}$ are listed. The accuracy of the estimated parameters has been characterised with the $T$ values, defined as the ratio of the parameter value over the estimated standard deviation of this parameter. For the kinetic parameters $k_{A B}$ and $Q_{i j}$ the $T$ values obtained are as large as $20-40$ for the chemisorption constants $Q_{i}$ in the range $3-10$.

The consumption rates of DNT are given by

$$
r_{A B}=k_{A B} \theta_{\mathrm{H}} \theta_{A} \text { and } r_{A D}=k_{A D} \theta_{\mathrm{H}} \theta_{A} \text {. }
$$

At the start of the reaction, the observed hydrogen

Table 6. Data on the hydrogen consumption rates (crushed catalyst, $d_{p}<40 \mu \mathrm{m}$ )

\begin{tabular}{|c|c|c|c|c|c|c|c|}
\hline Run & $\begin{array}{c}T \\
(\mathbf{K})\end{array}$ & $\begin{array}{c}p_{\mathbf{H}_{2}} \\
(\mathrm{kPa})\end{array}$ & $\begin{array}{l}m_{\text {cat }} \\
(g)\end{array}$ & $\begin{array}{c}m_{\mathbf{O}, \text { DNT }} \\
\quad(\mathrm{g})\end{array}$ & $\begin{array}{c}r_{0} \times 10^{3} \\
\left(s^{-1}\right)\end{array}$ & $\begin{array}{c}r_{\mathrm{o} . \mathrm{H}} \times 10^{3} \\
\left(\mathrm{~mol} \mathrm{~kg}^{-1} \mathrm{cat}^{-1} \mathrm{~s}^{-1}\right)\end{array}$ & $\begin{array}{c}c_{\mathrm{H}_{2 . \boldsymbol{\theta}}} \\
\left(\mathrm{mol} \mathrm{m}^{-3}\right)\end{array}$ \\
\hline $\begin{array}{l}\mathrm{C} 1 \\
\mathrm{C} 2 \\
\mathrm{C} 3 \\
\mathrm{C} 4\end{array}$ & $\begin{array}{l}308.5 \\
308.7 \\
308.6 \\
308.2\end{array}$ & $\begin{array}{r}492 \\
1000 \\
1974 \\
3888\end{array}$ & $\begin{array}{l}19.33 \\
19.87 \\
20.69 \\
10.41\end{array}$ & $\begin{array}{r}9.02 \\
8.99 \\
9.08 \\
10.02\end{array}$ & $\begin{array}{l}0.168 \\
0.242 \\
0.314 \\
0.159\end{array}$ & $\begin{array}{l}2.58 \\
3.61 \\
4.54 \\
5.04\end{array}$ & $\begin{array}{r}192 \\
390 \\
769 \\
1518\end{array}$ \\
\hline $\begin{array}{l}\text { C5 } \\
\text { C6 } \\
\text { C7 } \\
\text { C8 }\end{array}$ & $\begin{array}{l}345.6 \\
345.6 \\
346.0 \\
345.5\end{array}$ & $\begin{array}{r}497 \\
998 \\
2011 \\
3961\end{array}$ & $\begin{array}{l}19.93 \\
19.42 \\
18.96 \\
19.14\end{array}$ & $\begin{array}{l}9.59 \\
9.01 \\
9.10 \\
9.17\end{array}$ & $\begin{array}{l}1.22 \\
1.72 \\
2.41 \\
2.80\end{array}$ & $\begin{array}{l}18.1 \\
26.2 \\
38.1 \\
44.3\end{array}$ & $\begin{array}{r}173 \\
347 \\
699 \\
1379\end{array}$ \\
\hline
\end{tabular}


Table 7. Parameters for the intrinsic reaction kinetics

$$
T=308 \mathrm{~K}
$$

\begin{tabular}{|c|c|c|c|c|c|c|c|c|c|}
\hline$\left(\mathrm{mol} \mathrm{m}^{-3}\right)$ & $\begin{array}{c}k_{A B} \theta_{H} \times 10^{3} \\
\left(\mathrm{~mol} \mathrm{~kg}^{-1} \mathrm{~s}^{-1}\right)\end{array}$ & $a_{B C}$ & $q_{C E}$ & $\begin{array}{l}q_{A D} \\
\text { ension }\end{array}$ & $q_{D E}$ & $Q_{B}$ & $Q_{c}$ & $Q_{D}$ & $Q_{z}$ \\
\hline $\begin{array}{r}192 \\
390 \\
769 \\
1518\end{array}$ & $\begin{array}{l}1.04 \\
1.47 \\
1.77 \\
2.04\end{array}$ & $\begin{array}{l}3.32 \\
3.36 \\
3.23 \\
3.22\end{array}$ & $\begin{array}{l}0.16 \\
0.15 \\
0.15 \\
0.14\end{array}$ & $\begin{array}{l}0.14 \\
0.14 \\
0.16 \\
0.16\end{array}$ & $\begin{array}{l}3.56 \\
3.46 \\
3.95 \\
3.58\end{array}$ & $\begin{array}{l}0.11 \\
0.11 \\
0.11 \\
0.11\end{array}$ & $\begin{array}{l}0.70 \\
0.70 \\
0.72 \\
0.80\end{array}$ & $\begin{array}{l}0.036 \\
0.036 \\
0.035 \\
0.035\end{array}$ & $\begin{array}{l}0.001 \\
0.001 \\
0.001 \\
0.001\end{array}$ \\
\hline Average values & & 3.2 & 0.15 & 0.15 & 3.6 & 0.11 & 0.73 & 0.035 & 0.001 \\
\hline
\end{tabular}

\begin{tabular}{|c|c|c|c|c|c|c|c|c|c|}
\hline \multicolumn{10}{|c|}{$T=345 \mathrm{~K}$} \\
\hline $\begin{array}{r}173 \\
347 \\
699 \\
1379\end{array}$ & $\begin{array}{c}6.56 \\
8.83 \\
12.8 \\
14.0\end{array}$ & $\begin{array}{l}3.67 \\
3.63 \\
2.90 \\
2.93\end{array}$ & $\begin{array}{l}0.18 \\
0.14 \\
0.13 \\
0.15\end{array}$ & $\begin{array}{l}0.22 \\
0.23 \\
0.27 \\
0.28\end{array}$ & $\begin{array}{l}4.87 \\
4.73 \\
3.87 \\
3.98\end{array}$ & $\begin{array}{l}0.12 \\
0.11 \\
0.13 \\
0.13\end{array}$ & $\begin{array}{l}0.68 \\
0.66 \\
0.89 \\
0.89\end{array}$ & $\begin{array}{l}0.05 \\
0.05 \\
0.06 \\
0.06\end{array}$ & $\begin{array}{l}0.001 \\
0.001 \\
0.001 \\
0.001\end{array}$ \\
\hline Average values & & 3.6 & 0.13 & 0.26 & 4.3 & 0.12 & 0.78 & 0.05 & 0.001 \\
\hline
\end{tabular}

consumption rates are independent of the concentration of DNT. The Simusolv evaluations produce a value for $\theta_{A}$ close to unity up to a hydrogen conversion of 0.20 . Therefore, eq. (17) can be simplified and the initial rate of the hydrogen consumption can be found by

$$
r_{\mathrm{O}, \mathrm{H}}=r_{\mathrm{O}, A B}\left(2+3 q_{A D}\right)=k_{A B} \theta_{\mathrm{H}}\left(2+3 q_{A D}\right)
$$

The $r_{O, H}$ values as calculated from the Simusolv results have been compared to those obtained directly by linear regression of the experimental conversion curves. The data at $308 \mathrm{~K}$ are in excellent agreement and only for two runs, performed at $345 \mathrm{~K}$, a slight discrepancy is noticed.

Using eq. (18) the initial rates of conversion of $A \rightarrow B$ can be estimated- $\theta_{A}$ taken to be equal to $1-$ and evaluated according to

$$
r_{\mathrm{O}, A B}=k_{A B} \frac{K_{\mathrm{H}}\left(c_{\mathrm{H}_{2}, \theta}\right)^{n}}{1+K_{\mathrm{H}}\left(c_{\mathrm{H}_{2}, \theta}\right)^{n}}
$$

in which the parameter values of $k_{A B}$ and $K_{H}$ have been estimated for both temperatures separately with a non-linear regression method minimizing the sum of the squared residuals.

Note that the constant $K_{H}$ is a function of the true adsorption constant and the solubility of hydrogen in methanol. The parameters obtained and their temperature dependences for both molecular $(n=1)$ and dissociative adsorption $(n=0.5)$ models are presented in Table 8. On the basis of $T$ values, it is not possible to make a choice between adsorption models for hydrogen. In view of the work of Janssen et al. (1990b), we arbitrarily opt for the dissociative adsorption model.

The final results are collected in Table 9 as a function of temperature and are compared to those obtained by Janssen et al. (1990b) for the Pd/C catalyst. For $Q_{1}$ and $q_{C E}$ constant values are listed because a temperature dependence could not be determined

\begin{tabular}{|c|c|c|c|c|}
\hline \multicolumn{5}{|c|}{ (a) Molecular adsorption model, $n=1$} \\
\hline $\begin{array}{c}T \\
(\mathbf{K})\end{array}$ & $\left(\mathrm{mol} \mathrm{DNT} \mathrm{kg}^{k_{A B}}\right.$ cat s $\left.^{-1}\right)$ & $T_{k}$ & $\begin{array}{c}K_{\mathbf{H}} \\
\left(\mathbf{m}^{3} \mathbf{m o l}^{-1}\right)\end{array}$ & $T_{K}$ \\
\hline $\begin{array}{l}308 \\
345\end{array}$ & $\begin{array}{l}2.35 \times 10^{-3} \\
17.3 \times 10^{-3}\end{array}$ & $\begin{array}{r}>100 \\
53\end{array}$ & $\begin{array}{l}4.16 \times 10^{-3} \\
3.38 \times 10^{-3}\end{array}$ & $>\begin{array}{r}100 \\
14\end{array}$ \\
\hline \multicolumn{5}{|c|}{$k_{A \mathrm{~B}}=2.96 \times 10^{5} \exp \left(\frac{-5758}{T}\right)$} \\
\hline \multicolumn{5}{|c|}{ (b) Dissociative adsorption model, $n=0.5$} \\
\hline $\begin{array}{l}308 \\
345\end{array}$ & $\begin{array}{l}3.97 \times 10^{-3} \\
35.6 \times 10^{-3}\end{array}$ & $\begin{array}{l}44 \\
66\end{array}$ & $\begin{array}{l}28.0 \times 10^{-3 \dagger} \\
18.4 \times 10^{-3 \dagger}\end{array}$ & $\begin{array}{r}100 \\
26\end{array}$ \\
\hline & $3.23 \times 10^{6} \exp \left(\frac{-6334}{T}\right.$ & $K_{\mathbf{H}}=$ & $4 \times 10^{-4} \exp$ & \\
\hline
\end{tabular}
with a reasonable accuracy.

Table 8. Parameters estimated for crushed catalyst data, runs $\mathrm{C} 1-\mathrm{C} 8$

${ }^{+} K_{\mathrm{H}}$ in $\mathrm{m}^{3 / 2} \mathrm{~mol}^{-1 / 2}$. 
Table 9. Comparison of the parameters obtained for different types of the catalysts ${ }^{\dagger}$

\begin{tabular}{ccc}
\hline Parameter & This work: $\mathrm{Pd} / \mathrm{Al}_{2} \mathrm{O}_{3}$ crushed catalyst & Janssen et al. (1990b): Pd/C catalyst \\
\hline$k_{A B}$ & $3.23 \times 10^{6} \exp \left(\frac{-6330}{T}\right)$ & $1.2 \times 10^{9} \exp \left(\frac{-6850}{T}\right)$ \\
$K_{\mathrm{H}}$ & $5.5 \times 10^{-4} \exp \left(\frac{1210}{T}\right)$ & $4.3 \times 10^{-7} \exp \left(\frac{3630}{T}\right)$ \\
$q_{B C}$ & $8.9 \exp \left(\frac{-313}{T}\right)$ & $290 \exp \left(\frac{-1730}{T}\right)$ \\
$q_{C E}$ & 0.15 & $0.013 \exp \left(\frac{1160}{T}\right)$ \\
$q_{A D}$ & $25.6 \exp \left(\frac{-1586}{T}\right)$ & $2.3 \exp \left(\frac{-770}{T}\right)$ \\
$q_{D E}$ & $19.6 \exp \left(\frac{-520}{T}\right)$ & 1.1 \\
$Q_{B}$ & 0.11 & $645 \exp \left(\frac{-2800}{T}\right)$ \\
$Q_{C}$ & 0.76 & 0.13 \\
$Q_{D}$ & 0.04 & 0.06 \\
$Q_{E}$ & 0.001 & $1.9 \times 10^{-14} \exp \left(\frac{8854}{T}\right)$ \\
& &
\end{tabular}

${ }^{\top} \boldsymbol{K}_{A B}$ and $K_{\mathrm{H}}$ parameters are taken for a dissociative adsorption model, $n=0.5$

5.2. Kinetic modelling with internal diffusion limitations

The experimental hydrogen consumption rates for the crushed catalyst and for the pellets are presented in Fig. 10 as a function of the hydrogen conversion. These data have been obtained by point by point differentiating of the measured amount of hydrogen taken up by the system according to $r_{H}=$
$\left(-\Delta n_{\mathrm{H}_{2}} / \Delta t\right) / m_{\text {cat }}$, where $r_{\mathrm{H}}$ is expressed in moles of $\mathrm{H}_{2}$ consumed per unit mass of the catalyst per second. Observing our data at $308 \mathrm{~K}$, we see that pore diffusion resistances play a role in the initial region up to hydrogen conversions of about $0.3-0.5$. After that the reaction rate decreases, so internal diffusion resistances become less important and for $\zeta_{\mathrm{H}_{2}}$ greater than 0.6 the rate is so low that the catalyst efficiency is

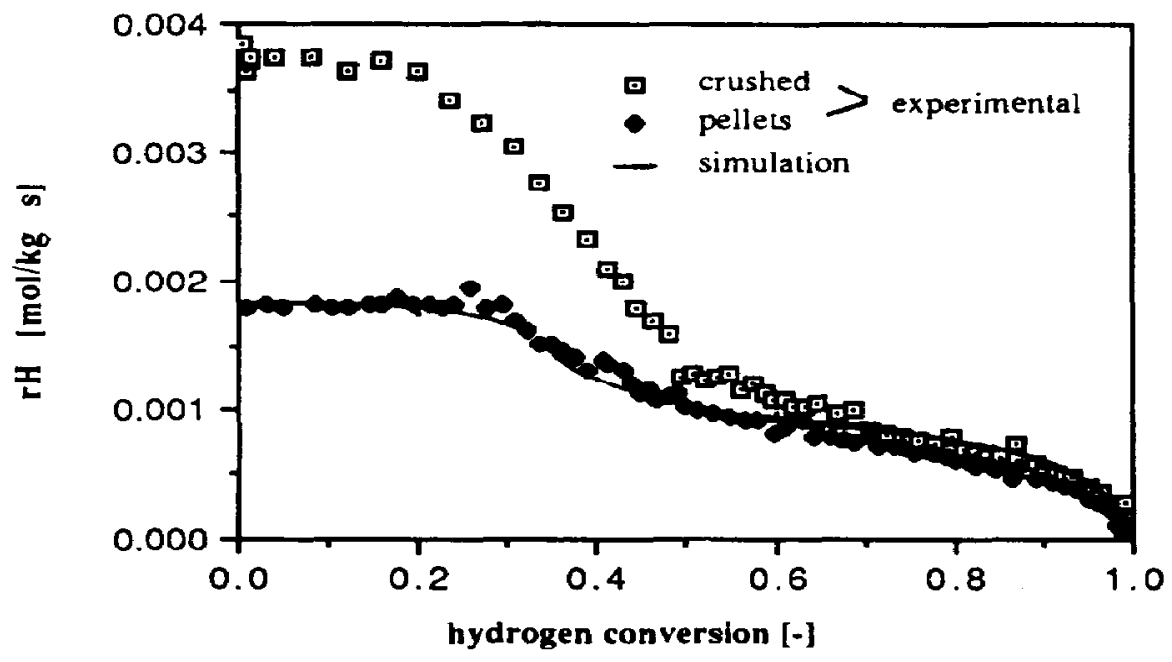

Fig. 10. Overall hydrogen consumption rates as a function of the hydrogen conversion for pellets and for crushed catalyst. $T=308 \mathrm{~K}, p_{\mathrm{H}_{2}}=1.0 \mathrm{MPa}, c_{\mathrm{AO}}=210 \mathrm{~mol} \mathrm{~m}^{-3}, m_{\mathrm{cat}}=20 \mathrm{~g}$. 
equal to one. At higher temperatures, the pore diffusion resistances will be significant for even higher values of $\zeta_{\mathrm{H}_{2}}$.

The significance of the internal diffusion limitations can be estimated directly in the initial period of the reaction where the overall hydrogen consumption rates are a function of the hydrogen concentration only. Because of the relatively large adsorption strength of DNT, local fractional coverages $\theta_{A}$ in the catalyst pores are close to unity in a wide range of molar concentrations of $c_{A}$. So, in the initial region we can assume that pore diffusion of hydrogen controls despite the fact that the diffusion coefficients of the organic compounds are lower than those for hydrogen (see Appendix). At higher conversions we keep assuming that only pore diffusion limitations of hydrogen and not of aromatic compounds play a role.

Since the penetration depth is much smaller than the pellet diameter, we can use a simple slab model with a penetration depth $\delta$ and a surface area equal to the pellet external surface area. Following Westerterp et al. (1987), a general equation for the effectiveness factor for a sufficiently fast reaction is given by

$$
\eta=\frac{1}{\delta R_{\mathrm{H}}\left(c_{\mathrm{His}}\right)} \sqrt{2 \int_{0}^{c_{\mathrm{H} t=}} R_{\mathrm{H}}(c) D_{\text {efr, } \mathrm{H}_{2}} \mathrm{~d} c}
$$

where $D_{\text {eff, } \mathrm{H}_{2}}$ is the effective diffusivity of hydrogen in the pores and $\boldsymbol{R}_{\mathrm{H}}$ is the reaction rate per unit volume of the catalyst layer. The hydrogen consumption rate $R_{H}$ in moles of $H_{2}$ per unit of active volume of the catalyst per second can be related to the overall initial rate $r_{H}$ in moles of $\mathbf{H}_{2}$ consumed per second per unit mass of the catalyst by

$$
R_{\mathrm{H}}=r_{\mathrm{H}} \rho_{\mathrm{cat}}\left(\frac{d}{6 \delta}\right)
$$

Expressing $\boldsymbol{r}_{\mathrm{H}}$ according to the reaction pathway, we obtain

$$
r_{\mathrm{H}}=2 r_{A B}+r_{B C}+3 r_{C E}+3 r_{A D}+3 r_{D E}=k_{o v} \theta_{\mathbf{H}}
$$

where the overall reaction rate constant equals

$$
\begin{aligned}
k_{o v}= & k_{A B}\left(2 \theta_{A}+q_{B C} \theta_{B}+3 q_{C E} \theta_{C}+3 q_{A D} \theta_{A}\right. \\
& \left.+3 q_{D E} \theta_{D}\right) .
\end{aligned}
$$

Note that in the initial region where $\theta_{A}=1$ and other $\theta_{i}$ are equal to zero, then

$$
k_{o v}=k_{0}=k_{A B}\left(2+3 q_{A D}\right) \text {. }
$$

After integration we obtain for molecular adsorption

$$
\begin{aligned}
& \eta=\frac{1+K_{\mathrm{H}} c_{\mathrm{H}_{2.8}}}{K_{\mathrm{H}_{1} c_{\mathrm{H}_{2}, g}}} \sqrt{\frac{2 D_{\mathrm{erf} . \mathrm{H}_{2}} c_{\mathrm{H}_{2.8} m} m}{\delta^{2} k_{\text {ov }} \rho_{\mathrm{cat}}} \frac{6 \delta}{d}} \\
& \times \sqrt{1-\frac{1}{K_{H_{1}} c_{\mathrm{H}_{2}, \theta}} \ln \left(1+K_{\mathrm{H}} c_{\mathrm{H}_{2,9}}\right)}
\end{aligned}
$$

and for dissociative adsorption

$$
\begin{aligned}
\eta= & \frac{1+K_{\mathrm{H}} \sqrt{c_{\mathrm{H}_{2}, g}}}{K_{\mathrm{H}} \sqrt{c_{\mathrm{H}_{2}, g}}} \sqrt{\frac{2 D_{\text {eff, }}, \mathrm{H}_{2} c_{\mathrm{H}_{2}, g} m}{\delta^{2} k_{o v} \rho_{\mathrm{cat}}} \frac{6 \delta}{d}} \\
& \times \sqrt{1-\frac{2}{K_{\mathrm{H}} \sqrt{c_{\mathrm{H}_{2}, g}}}+\frac{2}{K_{\mathrm{H}^{2} c_{\mathrm{H}_{2}, g}}^{2}} \ln \left(1+K_{\mathrm{H}} \sqrt{c_{\mathrm{H}_{2}, g}}\right)} .
\end{aligned}
$$

The solubility constant $m$ must be introduced into eqs (25) and (26) in order to obtain the liquid phase concentration of hydrogen. Meanwhile the factor $K_{H}$ is the product of the true adsorption constant and the solubility constant.

The experimental overall effectiveness factor at the start of the reaction can be estimated by

$$
\eta_{\mathrm{o}}=\frac{\left(r_{0, \mathrm{H}}\right)_{\text {pellets }}}{\left(r_{\mathrm{O}, \mathrm{H}}\right)_{\text {crushed }}} .
$$

\begin{tabular}{|c|c|c|c|}
\hline \multicolumn{4}{|c|}{$T=308 \mathrm{~K}$} \\
\hline $\begin{array}{c}c_{\mathrm{H}_{2.0}} \\
\left(\mathrm{~mol} \mathrm{~m}^{-3}\right)\end{array}$ & $\begin{array}{c}\left(k_{A B} \theta_{\mathrm{H}}\right) \times 10^{3} \\
\text { (from Simusolv) } \\
\left(\mathrm{mol} \mathrm{kg}^{-1} \mathrm{~s}^{-1}\right)\end{array}$ & $\begin{array}{c}\eta_{0, \text { exp }} \\
\text { (dimensionless) }\end{array}$ & $\begin{array}{c}\left(D_{\text {eff, } H_{2}} / \delta\right) \times 10^{s} \\
\left(\mathrm{~m} \mathrm{~s}^{-1}\right)\end{array}$ \\
\hline 193 & 0.59 & 0.52 & 2.7 \\
\hline 387 & 0.71 & 0.50 & 1.5 \\
\hline 778 & 1.15 & 0.64 & 1.5. \\
\hline 1535 & 1.55 & 0.76 & 1.3 \\
\hline \multicolumn{4}{|c|}{$T=345 \mathrm{~K}$} \\
\hline 175 & 1.38 & 0.23 & 3.3 \\
\hline 343 & 3.05 & 0.30 & 3.7 \\
\hline 693 & 5.26 & 0.33 & 2.8 \\
\hline 1387 & 7.06 & 0.40 & 2.5 \\
\hline
\end{tabular}

The effectiveness factors $\eta_{0}$ as calculated on the basis of the experimental data on the initial rate in Tables 3 and 6 are given in Table 10 as a function of the hydrogen concentration in the gas phase. We observe considerable internal diffusion resistances.

For the dissociative adsorption model the values of $D_{\text {eff, } \mathrm{H}_{2}} / \delta$ estimated from eq. (26) are listed in Table 10.

Table 10. Parameters obtained for catalyst pellets and initial overall effectiveness factors 
To this end, the overall initial rate constant $k_{0}$ and parameter $K_{H}$ as determined for the crushed catalyst have been taken. Using the correlation of Akgerman and Gainer (1972), we estimate the molecular diffusion coefficients for hydrogen dissolved in methanol to be $9.8 \times 10^{-9}$ and $16.3 \times 10^{-9} \mathrm{~m}^{2} \mathrm{~s}^{-1}$ at 308 and $345 \mathrm{~K}$, respectively. Using the temperature dependence of the tortuosity factor as given by Yücelen (1984), we take $\tau=3.6$ at $308 \mathrm{~K}$ and $\tau=4.0$ at $345 \mathrm{~K}$. For the experimentally determined porosity of the pellets of $\beta=0.46$ we obtain effective diffusion coefficients of $1.2 \times 10^{-9} \mathrm{~m}^{2} \mathrm{~s}^{-1}$ and $1.8 \times 10^{-9} \mathrm{~m}^{2} \mathrm{~s}^{-1}$ at 308 and $345 \mathrm{~K}$, respectively. Then the effective pore length $\delta$ is $63 \pm 5 \mu \mathrm{m}$.
For catalyst pellets the kinetic and chemisorption parameters have been estimated with the Simusolv program. Experimental data of the hydrogen conversion as well as the concentration data have been used. A comparison of the experimental results and simulation lines is given in Figs 11 and 12. A good agreement for the hydrogen conversion, with a maximal discrepancy of 0.05 absolute, can be observed. The concentration profiles for DNT, 2-A-4-NT, 4-A-2-NT and DAT are described with a smaller accuracy. This is caused mainly by analytical difficulties.

Calculations performed indicate that the kinetic and chemisorption parameters for pellets can be assumed to be the same within the confidence interval as

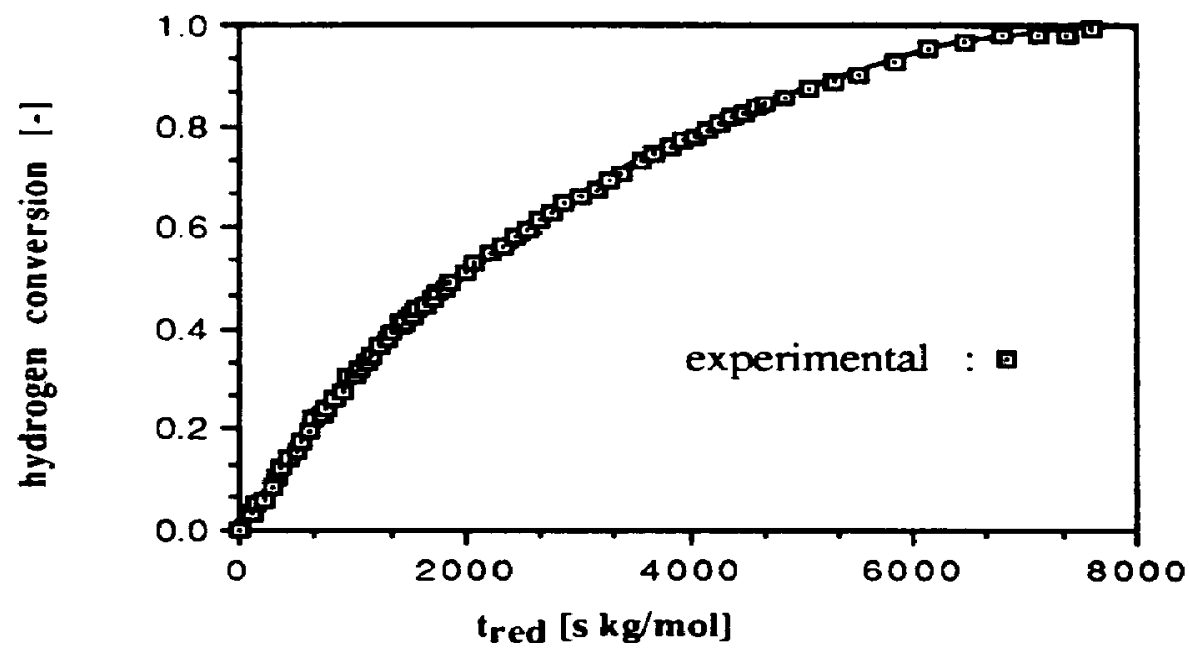

Fig. 11. Experimental hydrogen conversions and model predictions. $T=308 \mathrm{~K}, p_{\mathrm{H}_{2}}=1.0 \mathrm{MPa}$, $c_{A O}=219 \mathrm{~mol} \mathrm{~m}^{-3}, c_{\mathrm{cat}}=80 \mathrm{~kg} \mathrm{~m}^{-3}, N=25 \mathrm{rps}$, catalyst pellets.

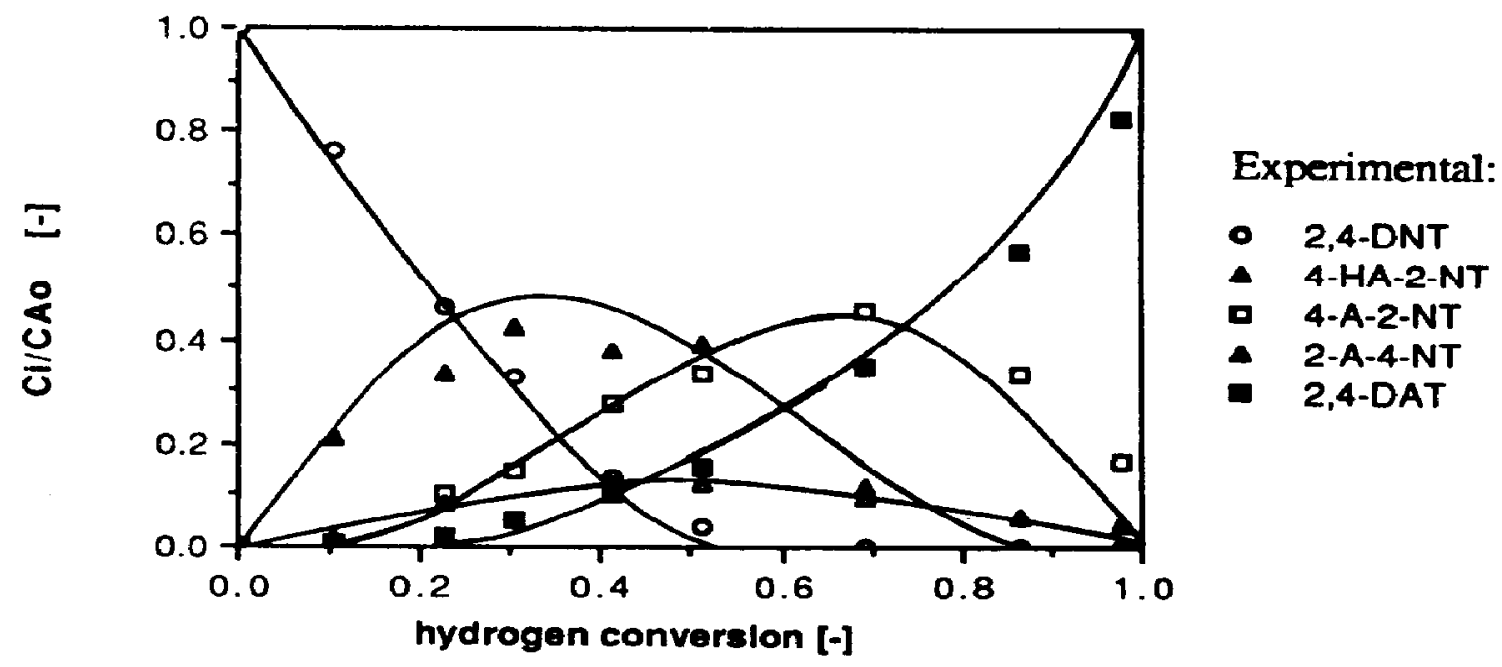

Fig. 12. Experimental concentration data for catalyst pellets and model predictions. $T=308 \mathrm{~K}$, $p_{\mathrm{H}_{2}}=1.0 \mathrm{MPa}, c_{A 0}=219 \mathrm{~mol} \mathrm{~m}^{-3}, c_{\mathrm{eat}}=80 \mathrm{~kg} \mathrm{~m}^{-3}, N=25 \mathrm{rps}$, catalyst pellets. 
Table 11. Comparison of the experimental and the calculated data at $371 \mathrm{~K}$

(a) Hydrogen consumption data for pellets

\begin{tabular}{|c|c|c|c|c|c|c|c|c|}
\hline Run & $\begin{array}{c}T \\
(\mathbf{K})\end{array}$ & $\left(\begin{array}{c}p_{\mathbf{H}_{2}} \\
(\mathbf{P P a})\end{array}\right.$ & $\left(\begin{array}{c}c_{\mathrm{H}_{2, g}}-3 \\
(\mathrm{~mol} \mathrm{~m}\end{array}\right.$ & $\begin{array}{c}m_{\mathrm{cat}} \\
(\mathrm{g})\end{array}$ & $\underset{(\mathrm{g})}{m_{\mathrm{ONT}}}$ & $\begin{array}{c}\left(r_{0 . H}\right)_{\operatorname{ern}} \times 10^{3} \\
\left(\mathrm{~mol} \mathrm{~kg}^{-1} \mathrm{~s}^{-1}\right)\end{array}$ & $\underset{\text { (dimensionless) }}{\boldsymbol{n}_{\text {o.cal }}}$ & 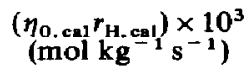 \\
\hline P9 & 371.6 & 493 & 160 & 18.04 & 9.51 & 6.5 & 0.15 & 7.8 \\
\hline P10 & 371.7 & 1046 & 338 & 17.91 & 9.52 & 14.6 & 0.18 & 14.8 \\
\hline P11 & 371.5 & 1974 & 639 & 18.03 & 9.50 & 25.6 & 0.22 & 24.2 \\
\hline P12 & 371.5 & 3590 & 1162 & 17.93 & 9.51 & 35.8 & 0.29 & 37.1 \\
\hline
\end{tabular}

(b) Concentration measurements for pellets at $p_{\mathrm{H}_{2}}=1.0 \mathrm{MPa}$ and for $c_{A 0}=105-215 \mathrm{~mol} \mathrm{~m}^{-3}$

\begin{tabular}{|c|c|c|c|c|c|c|c|c|c|}
\hline Run & $\zeta_{\mathrm{H}_{2}}$ & $C_{A}$ & $C_{\mathrm{B}}$ & $C_{c}$ & $C_{D}$ & $C_{E}$ & $\eta_{\mathrm{cal}}$ & $\left(\eta_{\text {cal }} r_{\mathrm{H}, \text { cal }}\right) \times 10^{3}$ & $\left(r_{H}\right)_{\exp } \times 10^{3}$ \\
\hline A14 & 0.179 & 0.738 & 0.000 & 0.148 & 0.074 & 0.075 & 0.19 & 14.0 & 14.6 \\
\hline A15 & 0.250 & 0.600 & 0.000 & 0.208 & 0.104 & 0.096 & 0.20 & 13.5 & 13.2 \\
\hline A 16 & 0.297 & 0.489 & 0.081 & 0.201 & 0.111 & 0.111 & 0.20 & 13.4 & 12.6 \\
\hline A 17 & 0.379 & 0.309 & 0.138 & 0.295 & 0.138 & 0.114 & 0.22 & 12.3 & 12.1 \\
\hline A19 & 0.385 & 0.385 & 0.025 & 0.295 & 0.131 & 0.164 & 0.22 & 12.3 & 12.1 \\
\hline A20 & 0.538 & 0.114 & 0.044 & 0.460 & 0.180 & 0.206 & 0.28 & 9.7 & 8.5 \\
\hline A21 & 0.696 & 0.005 & 0.000 & 0.449 & 0.157 & 0.403 & 0.38 & 7.2 & 6.0 \\
\hline
\end{tabular}

those for crushed catalyst. The influence of the pore resistance in the initial region is lumped in the values of product $k_{A B} \theta_{\mathbf{H}}$ as shown in Table 10 .

For an evaluation of the reaction rate of each reaction step the following expression is recommended:

$$
\begin{aligned}
r_{i j}= & k_{A B} q_{i j} \eta \frac{K_{\mathrm{H}} \sqrt{p_{\mathrm{H}_{2}} / R T}}{1+K_{\mathrm{H}} \sqrt{p_{\mathrm{H}_{2}} / R T}} \\
& \times \frac{Q_{i} C_{i}}{C_{A}+Q_{\mathrm{B}} C_{B}+Q_{C} C_{C}+Q_{D} C_{D}+Q_{E} C_{E}}
\end{aligned}
$$

where kinetic and chemisorption parameters $k_{A B}, q_{i j}, Q_{i}$ and $K_{H}$ are taken for intrinsic reaction kinetics as listed in Table 7 and the effectiveness factor $\eta$ is evaluated according to eq. (26). Note that in eq. (28) the parameters $Q_{A}$ and $q_{A B}$ are equal to unity and the concentrations of organic compounds are taken in the bulk of the liquid phase. The overall hydrogen conversion rates determined from eqs (28) and (22) are shown in Fig. 10 as a solid line. A good agreement with the experimental data is noticed.

\section{EXTRAPOLATION OF DERIVED RELATIONS}

Results are presented in the form of eqs (26) and (28), together with the relevant data given in Table 9. The relations enable us to predict conversion rates in the region of our experimental conditions.

In order to obtain an impression as to how far these data and correlations can be extrapolated, we have executed a series of measurements at $371 \mathrm{~K}$, of which the results are presented in Table 11 . These experimental results are now compared to the values predicted on the basis of eqs (26) and (28) and the data of Table 9. To this end, in eq. (26) we have substituted the following values: $D_{\text {eff, } \mathrm{H}_{2}}=2.2 \times 10^{-9} \mathrm{~m}^{2} \mathrm{~s}^{-1}, m$ $=0.173, \delta=63 \mu \mathrm{m}, d=4.2 \mathrm{~mm}$ and $\rho_{\text {cat }}=1590$ $\mathrm{kg} \mathrm{m}^{-3}$. In Table 11 (a) the calculated and the experimental initial rates are compared. We observe a good agreement between the data. After that we used eq. (26) to predict effectiveness factors beyond the initial region and compared the calculated and the experimental data at different conversion levels. Results are given in Table 11 (b). Again we observe a good agreement. We see that at least up to $371 \mathrm{~K}$ the derived rate and effectiveness expressions can be extrapolated and used over the entire conversion range. Also, it confirms our assumption that pore diffusion resistances for aromatic compounds do not play an important role.

\section{DISCUSSION AND CONCLUSIONS}

The temperature dependences of the kinetic and chemisorption parameters as well as the activation temperatures obtained in this work for the crushed $\mathrm{Pd} / \mathrm{Al}_{2} \mathrm{O}_{3}$ catalyst and those determined for $\mathrm{Pd} / \mathrm{C}$ catalyst by Janssen et al. (1990b) are compared in Table 9. Significant differences for the activation temperatures and frequency factors are noticed. The differences in the values of the frequency factors are caused by a different content of palladium in the catalysts and by different internal areas and pore sizes. The ratios of the rate constants $k_{A B}$ evaluated for the carbon carrier to those for the alumina carrier are in the range $65-80$, while the ratio of the palladium content is about 65 . Significant differences in the hydrogen adsorption heats can be noticed while comparing the expressions for $\boldsymbol{K}_{H}$ for both catalyst types. However, both expressions produce $K_{\mathrm{H}}$ values close to each other in the investigated temperature range. The larger data set of Janssen et al. (1990b) was obtained at three different temperatures and in the same equipment. We can therefore expect them to be more reliable. So, we have recalculated our data for 
the crushed catalyst fitting them to the kinetic expressions and the activation and chemisorption energies as found by Janssen et al. (1990b). The quality of the fit deteriorated and the sum of the squared residuals increased 4 to 9 times in comparison to our own correlations as determined. So, the activation and chemisorption energies for the carbon carrier are different from those for the alumina pellets. following conclusions:

-The catalytic hydrogenation of 2,4-dinitrotoluene ates have been found.

-Internal diffusion limitations play a role under our are used.

-The intrinsic reaction kinetics can be found for a higher than $40-60 \mu \mathrm{m}$. performance and selectivity obtained in a three-phase packed bubble column reactor using the palladium on alumina pellets as the catalyst.

Acknowledgement - This work was supported by the Netherlands Foundation for Chemical Research (SON). Dr Molga highly appreciates his research fellowship funded by SON. Mr A. Pleiter for his technical support and advice and $\mathrm{Mr} H$. J. Fontein for his assistance with the Simusolv program are gratcfully aknowledged.

$a$

$C_{i}$

c

$c_{\mathrm{H}_{2} \mathrm{~L}}$

$c_{\text {cat }}$

$c_{\mathbf{H} \text { is }}$

$c_{\mathrm{H}_{2}, g}$

$d$

$d_{p}$

D

$D_{\text {eff, } H_{2}}$

E

$k_{G}, k_{L}, k_{L S}$

$\boldsymbol{k}_{\boldsymbol{i j}}$

$k_{0}$
Our experiments and calculations lead us to the on $\mathrm{Pd} / \mathrm{Al}_{2} \mathrm{O}_{3}$ catalyst pellets proceeds via the same reaction pathways as elaborated by Janssen et al. (1990a) for Pd/C catalyst-three stable intermediexperimental conditions when the catalyst pellets finely crushed catalyst with particle diameters not

In a further study we will report on the reactor

\section{NO'TATION}

$\boldsymbol{k}_{\text {ov }}$

$\boldsymbol{K}_{\mathbf{i}}$

$J_{\mathrm{H}_{2}}$

$m$

$n_{\text {cat }}$

$M$

n

$N$

p

$q_{i j}$

$Q_{i}$

$r_{0}$

$\boldsymbol{r}_{\mathrm{D}, \mathrm{H}}$

$\boldsymbol{r}_{i j}$

$\boldsymbol{R}_{\boldsymbol{i j}}$

$\boldsymbol{R}_{\mathbf{H}}$

$\boldsymbol{R}_{\mathbf{H}_{2}}^{\prime \prime}$

$\boldsymbol{t}$

T

$\boldsymbol{T}_{\bar{i}}$

$V_{R L}$ moles $\mathrm{H}_{2}$ consumed per $\mathrm{kg}$ cat per second

overall rate constant, moles $\mathrm{H}_{2}$ consumed per $\mathbf{k g}$ cat per second

chemisorption constant, $\mathrm{m}^{3} \mathrm{~mol}^{-1}$ or $\mathrm{m}^{3 / 2} \mathrm{~mol}^{-1 / 2}$

hydrogen flux, mol m $\mathrm{m}^{-2} \mathrm{~s}^{-1}$

$c_{L} / c_{G}$, solubility constant, dimensionless

mass of the catalyst, $\mathbf{k g}$

expression given by eq. (7)

moles amount, mol

impeller speed, $s^{-1}$

pressure, $\mathbf{M P a}$

$k_{i j} / k_{A B}$, reduced rate constants, dimensionless

$K_{i} / K_{A}$, reduced adsorption constants, dimensionless

$\Delta \zeta_{\mathrm{H}_{2}} / \Delta t$, intial reaction rate, $\mathrm{s}^{-1}$

reduced initial reaction rate, moles $\mathrm{H}_{2}$ consumed per $\mathrm{kg}$ cat per second

conversion rate of reactant $i$ to $j$ per catalyst mass unit, moles converted per kg cat per second

conversion rate of reactant $i$ to $j$ per liquid volume unit, moles converted per $\mathrm{m}^{3}$ liq per second

hydrogen conversion rate per catalyst volume unit, $\mathrm{mol} \mathrm{m}^{-3} \mathrm{~s}^{-1}$

initial reaction rate per catalyst surface area unit, $\mathrm{mol} \mathrm{m} \mathrm{m}^{-2} \mathrm{~s}^{-1}$

time, $s$

temperature, $\mathrm{K}$

$T$ value for parameter $i$, dimensionless reactor liquid volume, $\mathrm{m}^{3}$

Greek letters

$\beta \quad$ internal pellet porosity, dimensionless

$\delta$

concentration, $\mathrm{mol} \mathrm{m}^{-3}$ or $\mathrm{kg} \mathrm{m}^{-3}$

$m p_{\mathrm{H}_{2}} / R T$, hydrogen equilibrium concen-

tration in the liquid phase, $\mathrm{mol} \mathrm{m}^{-3}$

concentration of the catalyst in the liquid

phase, $\mathbf{k g}$ cat $\mathbf{m}^{-3}$

hydrogen concentration at the catalyst

surface, $\mathrm{mol} \mathrm{\textrm {m } ^ { - 3 }}$

$p_{\mathrm{H}_{2}} / R T$, concentration of the hydrogen in

the gas phase, $\mathrm{mol} \mathrm{m}^{-3}$

diameter of a pellet, $m$

average particle diameter for the crushed

catalyst, $\mathbf{m}$

diffusion coefficient, $\mathrm{m}^{2} \mathrm{~s}^{-1}$

effective diffusivity of the hydrogen in the catalyst pores, $\mathrm{m}^{2} \mathrm{~s}^{-1}$

activation energy, $J \mathrm{~mol}^{-1}$

mass transfer coefficients, $\mathrm{m} \mathrm{s}^{-1}$

rate constant for the conversion of reactant $i$ to $j$, moles converted per $\mathrm{kg}$ cat per second

overall rate constant in the initial region,
$\zeta_{\mathbf{H}_{2}}$

$\eta$

$\boldsymbol{\theta}$

penetration depth, $m$

hydrogen conversion, dimensionless

overall effectiveness factor, dimensionless

fractional surface coverage, dimension-

less

$\tau$ tortuosity factor, dimensionless

$\rho_{\text {cat }} \quad$ density of catalyst pellets, $\mathrm{kg}$ per $\mathrm{m}^{3}$ cat

\section{Subscripts and superscripts}

$0 \quad$ initial

$\infty \quad$ final

cat catalyst

$G \quad$ gas

$\mathrm{H}_{2}, \mathrm{H} \quad$ hydrogen

$S \quad$ solid or surface

\section{Abbreviations}

A 2,4-DNT (2,4-dinitrotoluene)

B 4-HA-2-NT (4-hydroxylamino-2-nitrotoluene)

C 4-A-2-NT (4-amino-2-nitrotoluene)

D 2-A-4-NT (2-amino-4-nitrotolune)

E 2,4-DAT (2,4-diaminotoluene) red reduced

$L \quad$ liquid 


\section{REFERENCES}

Akgerman, A. and Gainer, J. L., 1972, Diffusion of gases in liquids. Ind. Engng Chem. Fundam. 11, 373-379.

Janssen, H. J., Kruithof, A. J., Steghuis, G. J. and Westerterp, K. R., 1990a, Kinetics of the catalytic hydrogenation of 2.4-dinitrotoluene. I. Experiments, reaction scheme and catalyst activity. Ind. Engng Chem. Res. 29, 754-766.

Janssen, H. J., Kruithof, A. J., Steghuis, G. J. and Westerterp, K. R., $1990 \mathrm{~b}$, Kinetics of the catalytic hydrogenation of 2,4-dinitrotoluene. II. Modelling of the reaction rates and catalyst activity. Ind. Engng Chem. Res. 29, 1822-1829.

Perry, R. H. and Chilton, C. H., 1974, Chemical Engineers' Handbook, 5th Edition, pp. 3-107. McGraw-Hill, New York.

Radhakrishnan, K., Ramachandran, P. A., Brahme, P. H. and Chaudhari, R. V., 1983, Solubility of hydrogen in methanol, nitrotoluene and their mixture. Experimental data and correlation. $J$. Chem. Engng Data 28, $1-4$.

Ramachandran, P. A. and Chaudari, R. V., 1983, Threephase catalytic reactors, in Topics in Chemical Engineering (Edited by Hughes, R.), Vol. 2. Gordon and Breach Service Publ., New York.

van Gelder K. B., Damhof, J. K., Kroijenga, P. J. and Westerterp, K. R., 1990a, Three-phase packed bed reactor with an evaporating solvent-I. Experimental: the hydrogenation of 2,4,6-trinitrotoluene in methanol. Chem. Engng Sci. 45, 3159-3170.

van Gelder K. B., Borman, P. C., Weenink, R. E. and Westerterp, K. R., 1990b, Three-phase packed bed reactor with an evaporating solvent-II. Modelling of the reactor. Chem. Engng Sci. 45, 3171-3192.

Westerterp, K. R., van Swaaij, W. P. M. and Beenackers, A. A. C. M., 1987, Chemical Reactor Design and Operation, 2nd Edition, pp. 433-436. Wiley, Chichester.

Westerterp, K. R., van Gelder K. B., Janssen, H. J. and Oyevaar, M. H., 1988, Development of catalytic hydrogenation reactors for the fine chemical industry. Chem. Engng Sci. 43, 2229-2236.

Yücelen, F., 1984, Effects of mass transfer in liquid phase consecutive hydrogenation of 2,6-dinitrotoluene, Ph.D. thesis, ETH Zurich.

Zwicky, J. J. and Gut, G., 1978, Kinetics, poisoning and mass transfer effects in liquid-phase hydrogenations of phenolic compounds over a palladium catalyst. Chem. Engng Sci. 33, $1363-1369$.

\section{APPENDIX}

The diffusion coefficients of organic compounds and of hydrogen in methanol have been estimated to be at $308 \mathrm{~K}$ equal to $2.0 \times 10^{-9} \mathrm{~m}^{2} \mathrm{~s}^{-1}$ and $9.8 \times 10^{-9} \mathrm{~m}^{2} \mathrm{~s}^{-1}$ for DNT and hydrogen, respectively. In the initial region, where DNT (A) is converted only into $B$ and $D$, the conversion rate of DNT can be described as follows:

$r_{A}=r_{A B}+r_{A D}=k_{A B} \theta_{\mathrm{H}} \theta_{A}+k_{A D} \theta_{\mathrm{H}} \theta_{A}=k_{A B}\left(1+q_{A D}\right) \theta_{\mathrm{H}} \theta_{A}$.

The initial overall rate of the hydrogen consumption can be found with

$$
r_{\mathrm{H}}=2 r_{A B}+3 r_{A D}=k_{A B}\left(2+3 q_{A D}\right) \theta_{\mathrm{H}} \theta_{A} .
$$

So the ratio of $r_{\mathrm{H}} / \boldsymbol{r}_{A}$ is equal to

$$
\frac{r_{H}}{r_{A}}=\frac{2+3 q_{A D}}{1+q_{A D}}
$$

This ratio is 2.1 and 2.2 at 308 and $345 \mathrm{~K}$, respectively; comparing with the values of the diffusion coefficients of hydrogen and DNT we can say that the molar concentration profile of DNT in the pores must be steeper than that for $\mathrm{H}_{2}$. Because of the non-linear dependence of the fractional coverages $\theta$ on the molar concentrations of reactants, it is not evident beforehand how local concentration gradients in the pores influence the reaction rate. An exact numerical solution for a parallel-consecutive reaction pathway with Langmuir-Hinshelwood kinetics and for pore diffusion of reactants playing a role is too complex for the moment. But some simple calculation tests can be made to predict which reactant controls the process in the initial region.

The sensitivities of $\theta_{H}$ and $\theta_{A}$ values on the possible pore concentration profiles of hydrogen and DNT, respectively, have been estimated.

The sensitivity

$$
\frac{\mathrm{d} \theta_{\mathrm{H}}}{\mathrm{d} c_{\mathrm{H} L}}=\frac{K_{\mathrm{H}}^{*}}{\left(1+K_{\mathrm{H}}^{*} c_{\mathrm{H} L}\right)^{2}}
$$

where $K_{H}^{*}$ is a true adsorption equilibrium constant and $c_{H L}$ a molar liquid concentration of hydrogen in pores, is a strong function of the concentration $c_{H L}$. It means that pore diffusion resistances of hydrogen play a larger role at a lower hydrogen pressure. This is confirmed experimentally (see Fig. A1). The dependence of $\theta_{A}$ on the local molecular con-

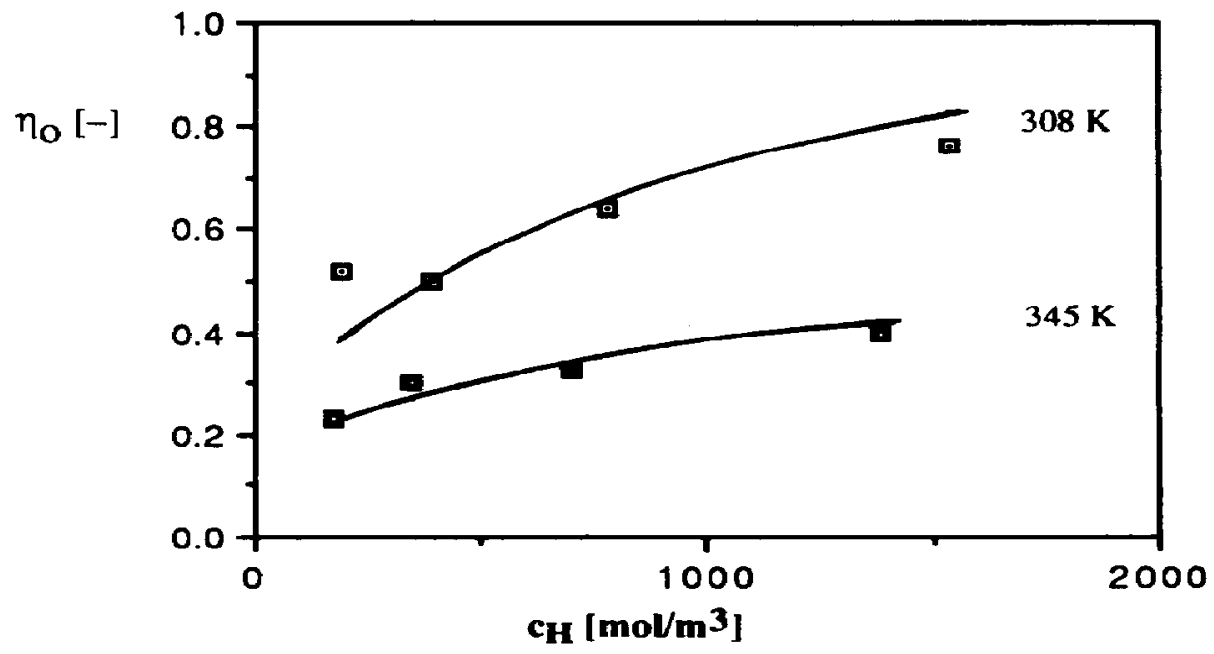

Fig. A1. An overall effectiveness factor in the initial region as a function of molar hydrogen concentration. 
centrations of DNT in the pores is more complex. Because the adsorption strength of DNT is high in relation to the other organic reactants, in the initial region local values of $\theta_{A}$ in the pores will be close to unity despite the concentration profile of $c_{A}$. The experimental data confirm this-if the fractional coverages $\theta_{A}$ in the pores would be smaller than unity, some active sites would be occupied by other organic species and as a result even in the initial region the intermediates $\mathrm{C}$ and/or final product $\mathrm{E}$ would be formed. However, in the initial region the reaction rate is constant (see Fig. 6) and component $C$ appears in the reaction mixture only after reaching a hydrogen conversion higher than 0.2 . 